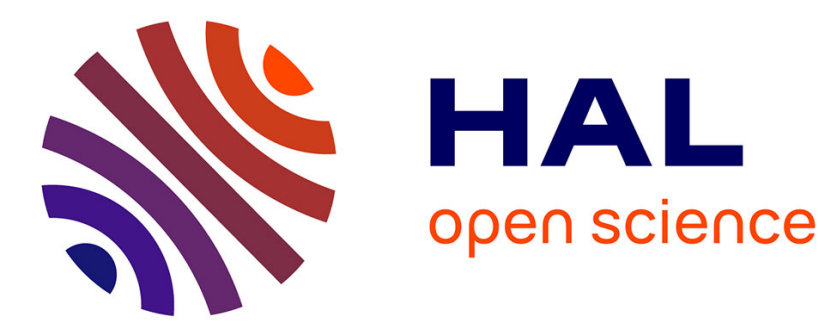

\title{
Singularity formation in vortex sheets and interfaces
}

Alberto Verga

\section{To cite this version:}

Alberto Verga. Singularity formation in vortex sheets and interfaces. Descalzi, Orazio and Martínez, Javier and Rica, Sergio. Instabilities and Nonequilibrium Structures IX, Kluwer, pp.361-388, 2004, NONLINEAR PHENOMENA AND COMPLEX SYSTEMS. hal-00092024

\section{HAL Id: hal-00092024 https://hal.science/hal-00092024}

Submitted on 8 Sep 2006

HAL is a multi-disciplinary open access archive for the deposit and dissemination of scientific research documents, whether they are published or not. The documents may come from teaching and research institutions in France or abroad, or from public or private research centers.
L'archive ouverte pluridisciplinaire HAL, est destinée au dépôt et à la diffusion de documents scientifiques de niveau recherche, publiés ou non, émanant des établissements d'enseignement et de recherche français ou étrangers, des laboratoires publics ou privés. 


\title{
Singularity formation in vortex sheets and interfaces
}

\author{
Alberto Verga, \\ Institut de Recherche sur les Phénomènes Hors Équilibre, \\ 49, rue F. Joliot-Curie, BP 146, 13384 Marseille, France
}

(Dated: July 26, 2002)

\begin{abstract}
One of the paradigms of nonlinear science is that patterns result from instability and bifurcation. However, another pathway is possible: self-similar evolution, singularity formation, and form. One example of this process is the formation of spherical drops through the pinch off of a cylindrical thread of liquid. Other example is given by the evolution of a vortex sheet, which from an initial regular shape, develops a finite time singularity of the curvature, resulting in the generation of a spiraling vortex. We investigate some simple systems, a stretched vortex sheet, the free surface of a perfect fluid driven by a vortex dipole, and the splash produced by a convergent capillary wave, in order to illustrate some specific scenarios to the appearance of a "form" through a singularity.
\end{abstract}

\section{INTRODUCTION}

The Escher lithograph "Print Gallery" [10] illustrates how a singularity can organize the surrounding space (figure 1). Indeed, in this print something impossible happens, something which cannot be drawn is there, masked at the center by the painter signature (a really unusual place to put it!). The center is a singularity, its presence allows strange phenomena to occur. Locally, all the details are coherent: a visitor with his hands behind his back, a woman looking down through her open window, the background town. However, globally one remarks that the young man on the left is simultaneously inside the gallery and outside, in the print he is seeing! The space is spiraling clockwise around the "empty center", as Escher describe it (figure 1). From a mathematical point of view this center is a singularity in an otherwise regular manifold (locally Euclidean): the field amplitude vanishes there but its phase turns around. What is relevant for us here is the ability of a singularity to determine, globally, the system's properties. The structure itself of the singularity in the Escher print can be deduced from the observation that any characteristic length scale can be identified. Indeed, the object sizes growth from the center, length scales increase following a spiral from the center. Therefore, following a counter-clockwise direction one arrives at the conclusion that at the center the scale length must vanish: this is a self-similar effect, and the result of this self-similarity is the appearance of a singularity. The object of this work is to illustrate, using simple physical systems, the relation between similarity, singularity and form.

*Electronic address: Alberto.Verga@irphe.univ-mrs.fr

†UMR 6594, Université d'Aix-Marseille, CNRS. 


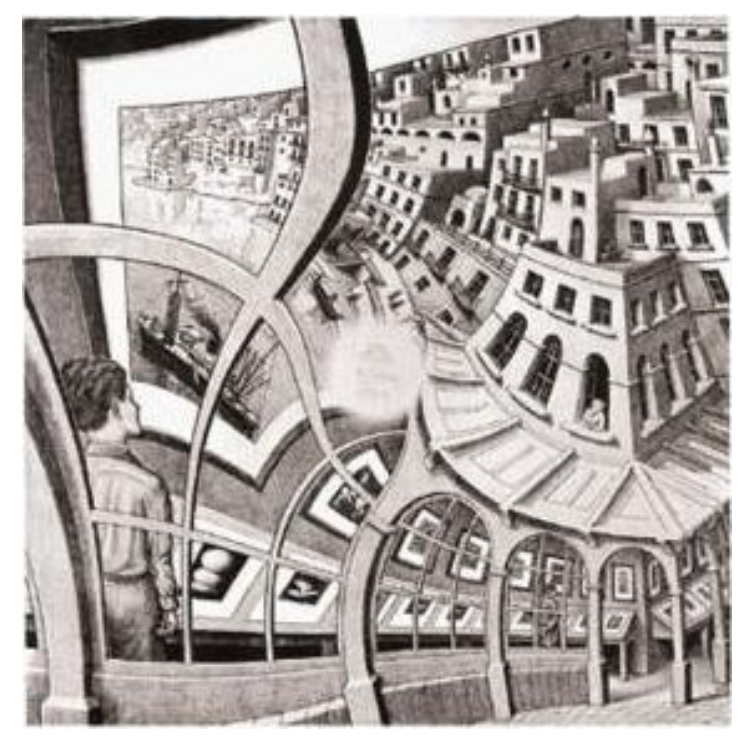

FIG. 1: Escher lithograph "Print Gallery", 1956, $32 \times 32 \mathrm{~cm}^{2}$ : "The boy sees all these things as twodimensional details of the print that he is studying. If his eye explores the surface further then he sees himself as part of the print." [10]

\section{A. Patterns and topological transitions}

Pattern formation is usually related to the existence of an instability of the basic homogeneous state, or, in more complicated situations, to the instability of more complex states (oscillating, spatially periodic, etc.). This instability appears at some definite value (perhaps zero) of an order parameter, whose variation may drive the system to different regimes, through successive bifurcations. The susceptibility of the system manifests also in the fact that small variations of the external parameters, that control its state, can result in drastic changes (bifurcation) in its physical properties, such as symmetry breaking transitions. As a consequence of the instability some characteristic length (and time), appears in the physical system, and a pattern forms. Often the selection of characteristic scales results from the interplay of linear and (weak) nonlinear effects, that depend essentially on the type of bifurcation encountered. This mechanism is reminiscent of phase transitions, when the temperature changes, intrinsic symmetries of the system may appear (or disappear), like the formation of a crystal structure from the isotropic liquid state. However, bifurcation, instability and form is not the only pathway by which a physical system can create intrinsic length or time scales: there exist purely dynamical mechanisms at the origin of morphogenesis. These dynamical mechanisms are not directly related to some external change of an order parameter, but, on the contrary, in a manner independent of external constraints, the system is able to generate its own length and time scales at the origin of form.

An example may clarify these statements. Let us consider the wake behind a body, produced by the flow of some liquid around it. If the fluid flow has a characteristic velocity $U$ and kinematic viscosity $\nu$, and the body size is $L$, one can form a Reynolds number $\operatorname{Re}=U L / \nu$. At low $\operatorname{Re}$ the flow is laminar, but at some critical value $\mathrm{Re}_{0}$ a recirculation zone appears, and for Reynolds numbers larger than some other critical value $\mathrm{Re}_{1}$, a periodic release of vortices is observed. One 
may interpret $\mathrm{Re}_{0}$ as the transition point between a homogeneous state and an inhomogeneous one, and $\mathrm{Re}_{1}$ as signaling a Hopf bifurcation point, through which the system losses its stationarity. The mathematical description of these bifurcations is given by the Ginzburg-Landau equation of some amplitude $A$, whose spatial and temporal variation describes the spatial and temporal pattern of the wake. However, there are other features of the wake formation that cannot be described in terms of weak nonlinear phenomena. These effects appear clearly if one considers the same problem, but as an initial value one: instead of changing the control parameters, one left the system evolve from an initial state. The transitions between different states are in fact accompanied by topological changes in the flow structure. Indeed, the appearance of a recirculation, from an initial laminar state for instance, is only possible if a stagnation point (a point with zero velocity) is formed inside the fluid (and not only on the body wall). Analogously, the state of periodic release of vortices establishes only after some transitory, and it implies a complex separation mechanism of vorticity from the body walls. The important point is that in these cases, the system evolution entails some singular behavior. To see this it is better to consider the perfect fluid limit. In this limit the flow topology is conserved. Therefore, the above effects, appearance of a recirculation zone, or the release of vortices, are only possible if at some moment the initially regular velocity field undergoes a singularity allowing the topological change to occur. Regularization by viscosity does not fundamentally change these statements, because the length and time scales resulting from the transition are not directly related to viscous effects. In summary, the transition between two distinct topologies can be described, not only in terms of bifurcation, but also in terms of "finite time" singularities. Instead of changing external parameters to pass from a state to another one, the system can evolve self-similarly from an initial (perhaps unstable) state with a given topology, towards a topologically different state through a singularity.

\section{B. Topological change in a Hele-Shaw interface}

In order to fix these ideas we study a simple physical system amenable to an exact computation of the topological transition: the Hele-Shaw flow of an interface between a non-viscous fluid (air) and a viscous one (oil). The fluids are confined in the gap of width $b$, between two parallel plates. The oil velocity $\mathbf{v}$ is given by the Darcy law. The fluid is driven by the pressure gradient $\mathbf{v}=-\left(b^{2} / 12 \mu\right) \nabla p$, where $\mu$ is the oil viscosity (the viscosity of the air is neglected). From the incompressibility condition $\nabla \cdot \mathbf{v}=0$ one finds that the pressure $p=p(x, y, t)$ is harmonic

$$
\Delta p(x, y, t)=0, \quad \text { in } \mathcal{D},
$$

in the more viscous fluid domain $\mathcal{D}$. The air-oil interface $\mathcal{C}$ is determined from de condition of mechanical equilibrium, it is a line of constant pressure $p=0$ (we neglect the effects of surface tension), and the condition of mass conservation, or in other terms, the advection of the constant pressure line with the fluid velocity:

$$
p_{t}+\mathbf{v} \cdot \nabla p=p_{t}-\left(b^{2} / 12 \mu\right)|\nabla p|^{2}=0, \quad \text { at } \mathcal{C},
$$

where the index $t$ means time derivative (in general we denote derivatives with subscript variables). The relation (1), indicates that the normal velocity of the interface is $V_{n}=-\partial_{n} p$, where $\partial_{n}$ is the gradient in the normal direction (at the interface). It is convenient to choose the length unit $b$, 
time unit $b^{2} \rho / 12 \mu$ and mass unit $\rho b^{3}$ ( $\rho$ is the constant viscous fluid density) in order to eliminate non-essential parameters. In this unit system the above equations reduce to

$$
\begin{cases}\Delta p=0, & \text { in } \mathcal{D}(t), \\ p=0, p_{t}-|\nabla p|^{2}=0, & \text { at } \mathcal{C}(t),\end{cases}
$$

valid at the interface $\mathcal{C}(t)$, boundary of the fluid domain $\mathcal{D}(t)$, where $p(x, y, t)=0$ can be considered as its implicit function equation. The Hele-Shaw problem entails to find the harmonic function $p$ in $\mathcal{D}(t)$ on the moving boundary $\mathcal{C}(t)$ which is itself an unknown satisfying the last equation in $(2)$. The initial shape of the interface is given by $\mathcal{C}(0): p(x, y, 0)=0$.

In order to simplify this problem we introduce the complex velocity potential $f(z)=-p+\mathrm{i} \psi$ and make a conformal transformation $z=z(t, \psi)$ such that, in the complex $\psi$ plane the interface has the simple equation $\Im \psi=0$ (independent of time). Here $\psi$ is a current function ( $p$ is equivalent to a velocity potential), and the original fluid domain is transformed to the $\Im \psi<0$ semi-plane. Using this analogy with the perfect fluid flow, it is clear that the normal velocity at the interface is given by $V_{n}=-\partial p=\partial_{l} \psi$, where $\partial_{l}$ is the tangential derivative and $l$ the arclength. Moreover, we have the geometrical definition $V_{n}=\Im\left(\bar{z}_{t} z_{l}\right)(z=z(t, \psi)$ is the parametric equation of a curve in the plane, with parameter $\psi)$. Therefore, the form of the interface is determined by the equation $\Im\left(\bar{z}_{t} z_{l}\right)=\partial_{l} \psi$, or

$$
\Im\left(\bar{z}_{t} z_{\psi}\right)=1
$$

where $\psi$ is taken at the interface $\Im \psi=0$. This is the well known Laplacian growth equation first derived to describe solidification fronts and dendritic growth, but appearing also in the problem of surface waves in a perfect fluid [32]. This equation has families of solutions including finite time singularities. Most of these solutions are regularized by an arbitrary small surface tension and are therefore not physically relevant. However, some particular singular solutions may have a signification even in the presence of small surface tension.

We study the formation of a drop from an initial flat interface through a "pole" solution of the Laplace growth equation. This solution has the peculiar property that it can be followed even after the formation of a singularity (singularity associated with the topological transition). The form of the conformal transformation (or equivalently of the parametric curve defining the interface) is assumed to be

$$
z(t, \psi)=\psi-\mathrm{i} t+\frac{a(t)}{\psi-\mathrm{i} b(t)},
$$

where $a(t)$ and $b(t)$ are two real functions of time to be determined by substitution of (4) into (3). This substitution give the two differential equations:

$$
\begin{aligned}
b \dot{a}+a \dot{b}+a & =0, \\
b\left(a+b^{2}\right) \dot{a}-a\left(a+b^{2}\right) \dot{b}-a b^{2} & =0 .
\end{aligned}
$$

Formula (4) together with $a(t)$ and $b(t)$ satisfying (6) give the form of the interface in time. A typical solution of these equations is depicted in figure 2. The initial flat interface develops a finger while advancing; this finger progressively shrinks at its base, up to finally form a separated drop of fluid. We note that both functions $a(t)$ and $b(t)$, tend to zero simultaneously, at a time $t=t_{*} \approx 1.62$. An exact particular solution of the system $(6)$ can be found in terms of powers

$$
\left\{a(t)=-4\left(t_{0}-t\right)^{2} / 9, b(t)=\left(t_{0}-t\right) / 3\right\},
$$



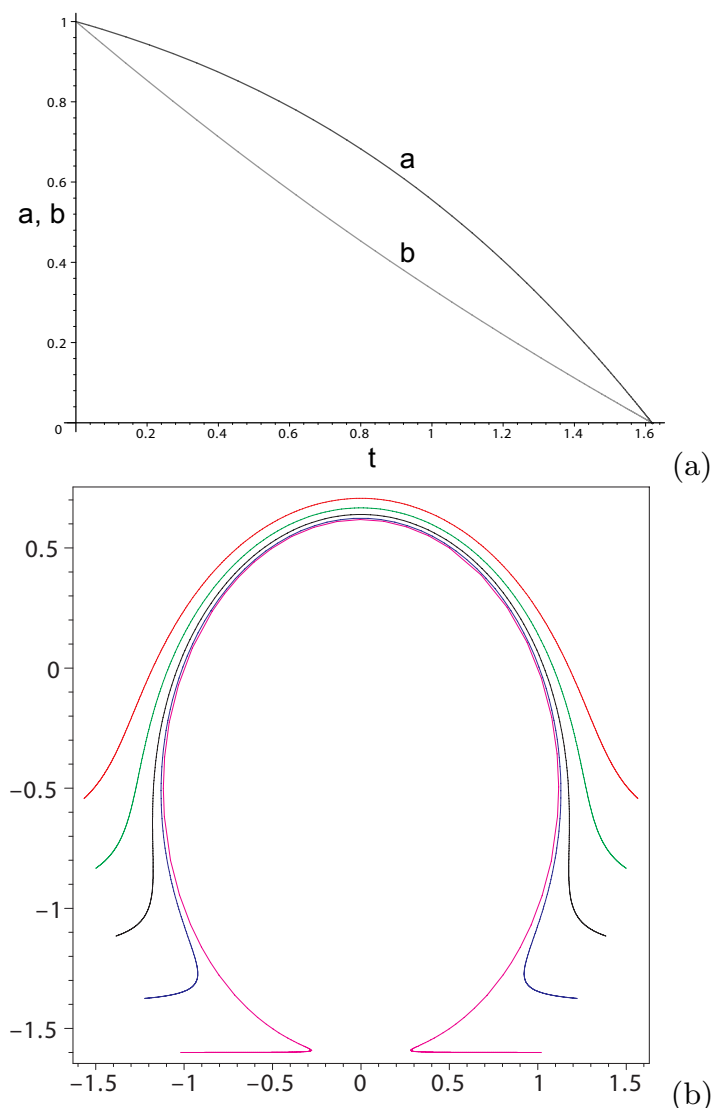

(a)

(b)

FIG. 2: Typical behavior of the pole coefficients $a(t)$ and $b(t)$ of formulas (6) in the solution of the HeleShow interface evolution (a). Both functions vanish linearly at the same critical time $\left(t=t_{*} \approx 1.62\right)$. Form of the interface at different times, $t=0.8: .2: 1.6$ (b). For increasing time $a$ and $b$ decrease, the interface shrinks, and tends to form two symmetric cusps at the moment where a drop separates.

where $t_{0}$ is an arbitrary parameter. This solution describes the behavior of $a(t)$ and $b(t)$ for a certain range of times before the time of the singularity formation (the separation of the drop), but not in the very neighborhood of the singularity. Near the singularity $a(t)$ and $b(t)$ can be developed in powers of $t_{*}-t$, where $t_{*}$ denotes the singularity formation time, to obtain,

$$
\left\{a(t)=A\left(t_{*}-t\right), b(t)=\left(t_{*}-t\right) / 2\right\},
$$

where $A$ is a free parameter (determined by the external solution). Therefore, the approach of the two sides of the interface towards the point of contact, follows a power law in time; these two points $\left( \pm x_{*}, y_{*}\right)$, having vertical slope, approach the singularity at a velocity diverging as

$$
\frac{d x_{*}}{d t}=\sqrt{A /\left(t_{*}-t\right)}, \quad \text { for } t \rightarrow t_{*} .
$$

These formulae describe completely the behavior of the interface up to the moment of its selfintersection. Moreover, the fact that $a(t)$ and $b(t)$ vanish at the same time, means that (4) remains analytic up to the time of contact, the pole contribution vanishes at just this contact instant, and therefore, the conformal transformation can be continued for times $t>t_{*}$ after the singularity by 
simply adding the front line $\mathcal{C}_{1}$ and the trailing circle $\mathcal{C}_{2}$ :

$$
z(t, \psi)= \begin{cases}\psi-\mathrm{i} t & \text { at } \mathcal{C}_{1}(t), \\ \left(a\left(t_{*}\right) / b\left(t_{*}\right)\right) \mathrm{e}^{2 \pi \mathrm{i} \psi}+\mathrm{i} y_{*} & \text { at } \mathcal{C}_{2} .\end{cases}
$$

The important fact about (10) is the appearance of a new scale in the system, the circle radius $a\left(t_{*}\right) / b\left(t_{*}\right)$ (although $a$ and $b$ vanish at $t_{*}$, their ratio is finite, as given by (8)). This length scale depends on the nature of the singularity (it depends on the singularity time $t_{*}$ through the functions $a, b$ ), and its appearance is not a straightforward consequence of the initial condition.

Therefore, in this example issued form the theory of the flow in a Hele-Shaw cell, in the absence of capillary effects (which in fact regularize the system's evolution), one can follow the emergence of (i) a self-similar evolution, leading to (ii) a finite time singularity associated to a change in the topology of the interface, and the subsequent introduction of (iii) a new scale, dynamically determined, signaling the formation of a spatial structure. This three steps scenario, similarity, singularity and form, of "pattern formation", does not need to invoke the variation of an external parameter in order to describe the emergence of new characteristic scales or topological changes, and as a consequence, it is worth interesting to be investigated in different physical systems. In the following sections we briefly describe some of these mechanisms at work in the context of vortex sheets and fluid interfaces.

\section{VORTEX SHEETS: INSTABILITY AND BREAKDOWN}

A vortex sheet (see figure 3) is, in a perfect fluid, a tangential velocity discontinuity: the vorticity is concentrated in a surface rather than in volume. In real fluids, possessing a finite viscosity, these sheets have a small width and are commonly created by the motion of bodies having sharp edges. They may also spontaneously appear in a strong shear flow or in shear layers. The classical example is the flow around a delta wing, where the boundary layer vorticity separates at the wing edge forming the vortex sheet. This vortex sheet rolls-up to form a spiraling conical surface and, by vorticity diffusion near its center, it evolves into a strong vortex. Shear layers are also common in fluid flows, and it is not too hard to see the characteristic cloud patterns resulting from the Kelvin-Helmholtz instability in the stratified atmosphere.

Figure 3 shows the rolling-up of a vortex sheet generated by the circular motion of a rectangular plate immersed in water, having a sharp edge (of dihedral angle $\pi / 6$ ). Vorticity is visualized by a fluorescent colorant painted on the plate edge, and lighted by a laser on a plane perpendicular to the plate axis. A camera is disposed under the tank containing the fluid. The sequence follows in time the formation of a characteristic spiral, where the vorticity is concentrated. The Reynolds number of the flow in this experiment is $\mathrm{Re}=17500$ and the angular motion of the plate is uniformly accelerated one (the plate angular velocity is proportional to time, $\Omega \propto t$ ). Small undulations grow on the spiral, forming a succession of secondary vortices. The origin of these secondary vortices is not so clear, in principle they may grow from spurious perturbations driven by defects in the plate motion, or on the contrary, they can be the manifestation of an intrinsic instability of the vortex sheet roll-up. In this section we study this secondary instability and the subsequent non-linear evolution of the perturbations. 

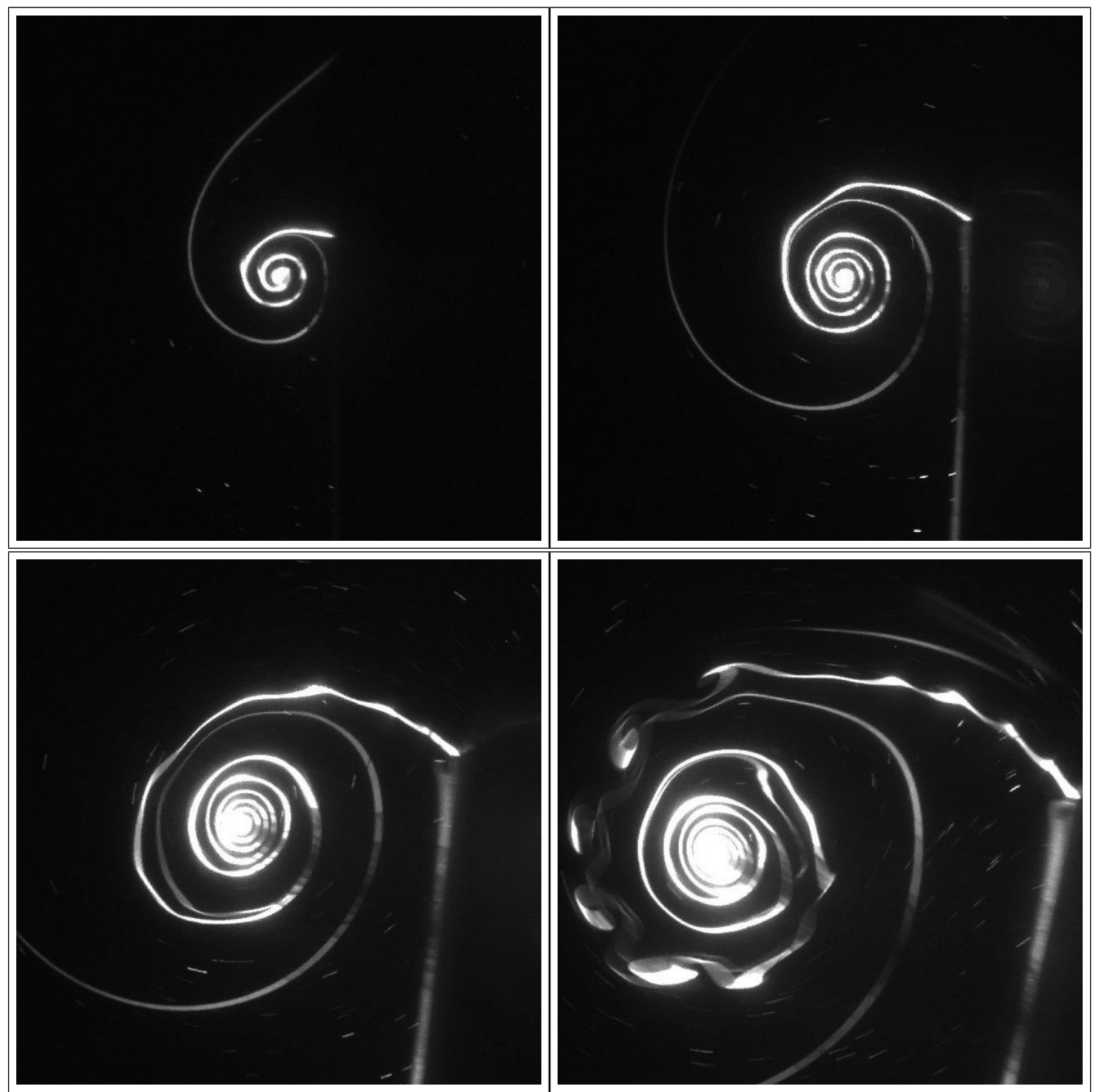

FIG. 3: Experimental vortex sheet rolling up. The four pictures (from left to right and from top to bottom) represent the spiral sheet at times $0.3,0.6,0.9$ and 1.2 , respectively. Vortex sheet instability generates secondary vortices which modify the main vortex structure (bottom right panel).

\section{A. Vortex sheet instability and Moore singularity}

In two dimensional perfect fluid flows a compact description of the vortex sheet dynamics exists, based on the Birkhoff-Rott equation [26],

$$
\frac{\partial}{\partial t} \overline{z(\Gamma, t)}=\frac{-\mathrm{i}}{2 \pi} f \frac{d \Gamma^{\prime}}{z(\Gamma, t)-z\left(\Gamma^{\prime}, t\right)},
$$


where $z(\Gamma, t)=x(\Gamma, t)+\mathrm{i} y(\Gamma, t)$ is the complex coordinate of a point on the vortex sheet, the circulation $\Gamma$ satisfying the relation

$$
\frac{d \Gamma}{d t}=0
$$

is an appropriate parameter of the curve (a vortex sheet in a two dimensional flow is then represented as a line in the complex plane). The integral in (11) is computed over the whole vortex sheet (the range of $\Gamma$ ), and must be interpreted in the sense of the Cauchy principal value. As a consequence of the Helmholtz theorem on the conservation of circulation, the vorticity initially concentrated on the sheet remains on there for later times. The velocity field, which is potential everywhere outside the sheet itself, can be computed directly (through the Biot-Savart equation) from the knowledge of the sheet motion. Therefore, the solution of the Euler equation reduce to the solution of the initial value problem (11).

The Birkhoff-Rott equation is also a good starting point to devise numerical algorithms. A simple discretization of the integral gives a set of differential equations analogous to the motion of point vortices: the vortex sheet can be thought as the continuous limit of a succession of point vortices. However, direct discretization is not possible due to the singular nature of the kernel (related to the Green function of the Laplace operator), and some regularization must be introduced. Krasny [15] proposed to modify the kernel in (11) in order to smooth out the smallest scales (related to the discretization method) by introducing a cut-off parameter $\delta$, that regularize the short distance interaction of point vortices,

$$
\frac{1}{z} \rightarrow \frac{\bar{z}}{|z|^{2}+\delta^{2}}
$$

this prescription is also compatible with the Cauchy principal value of the original equation (11), in the limit $\delta \rightarrow 0$, the pole contribution, with $\delta$ in the numerator, being excluded.

The simplest vortex sheet is a plane $z(\Gamma)=\Gamma / U$ of constant tangential velocity discontinuity $U \equiv U / 2-(-U / 2)$. A plane vortex sheet is an exact solution of the Birkhoff-Rott equation, but it is sensitive to the Kelvin-Helmholtz instability. Indeed, a perturbation of wavelength $\lambda$ and amplitude $\epsilon$, proportional to $\epsilon \sin (2 \pi \Gamma / \lambda U)$, or

$$
z(\Gamma, 0)=\Gamma+\epsilon(1-\mathrm{i}) \sin (2 \pi \Gamma)
$$

where lengths are measured in units of $\lambda$ and velocities in units of the vortex sheet strength $U$, is unstable as can easily be verified by linearizing (11) around the plane basic state $\epsilon=0$. A simple dimensional argument permits to write the growth rate $\sigma \propto U / \lambda$ as a function of the dimensional parameters $\lambda$ and $U$. A complete calculation gives $\sigma=\pi U / \lambda$, or for an arbitrary wavenumber the growth rate is found to be

$$
\sigma(k)=k / 2
$$

(in non-dimensional quantities). Equation (15) shows that the linearized Birkhoff-Rott equation is ill posed: even if initially the shape of the vortex sheet is regular, the Fourier modes increase exponentially with $k$ after a finite time, reflecting the appearance of a singularity. This situation is similar to the one encountered in the Hele-Shaw problem, with the Laplace growth equation (3). It is straightforward to show that the plane front $z(\psi, t)=\psi-$ it is unstable, and that the growth rate of this instability is also proportional to $|k|$. Non-linear interactions are not able to render the 
interface smooth for all times, and finite time singularities arise during its evolution. Therefore, one important question arises about the ill-posedness of the vortex sheet motion, as is actually the case for the interface propagation in a Hele-Show cell: one may ask whether non-linear terms will regularize the evolution. This question was thoroughly analyzed by Moore in a celebrated paper [22]. He found that the Kelvin-Helmholtz instability leads to a finite time singularity, the vortex sheet curvature diverges at some critical time $t_{*}$. Although not yet demonstrated, one may speculate that this curvature singularity is the seed of the spiral which rolls-up in the compression region of the vortex sheet (the region where proper lengths shrink).

From the physical point of view the appearance of a singularity during the nonlinear evolution of the Kelvin-Helmholtz instability can be related to the unavoidable existence of a topological transition between the initial state of a plane sheet and the subsequent development of another state dominated be a spiral rolling-up of the sheet. These spirals, arising from the growth of a Kelvin-Helmholtz instability, are commonly seen in natural shear layers, or in direct numerical simulations of the regularized Birkhoff-Rott equation $[15,17]$. Indeed, the transition from a line (the initial vortex sheet is a continuous deformation of a straight line) and a double branched spiral is impossible without the introduction of a new scale: the curvature radius of the spiral at its center. This length must vanish when the width of the vortex sheet goes to zero, the Euler case. Therefore, in the limit of an infinitely thin layer one must admit a divergence of the curvature as the necessary precursor of the spiral.

From the mathematical point of view, the singularity is related to the collision of a pole in the complex $\Gamma$ plane (the "length" parameter of the curve) to the real axis, in a finite time. The first step to demonstrate the mechanism of singularity formation is to analytically continue the BirkhoffRott equation to the upper half complex $\Gamma$ plane (a similar computation can be done to the lower half complex plane). The form of the vortex sheet is given by the function $z(\Gamma, t)=\Gamma+s(\Gamma, t)$, with $s(\Gamma+2 \pi)=s(\Gamma)$ and $s(-\Gamma)=s(\Gamma)$ (periodic, odd perturbation of the planar sheet). Introducing the operator ${ }^{*}$, to generalize the complex conjugation: $s^{*}=\overline{s(\bar{\Gamma})}$, one sees that for real $\Gamma$ the property $s^{*}=\bar{s}$ allows to perform the analytical continuation:

$$
s_{t}^{*}(\Gamma, t)=\frac{-\mathrm{i}}{2 \pi} f \frac{d \Gamma^{\prime}}{\Gamma-\Gamma^{\prime}+s(\Gamma, t)-s\left(\Gamma^{\prime}, t\right)},
$$

if $s$ is analytic (see refs. $[7,8]$ ). By simple manipulation of (16) one can transform the principal value integral into a contour integral plus the pole $\Gamma=\Gamma^{\prime}$ contribution,

$$
\begin{aligned}
f \frac{d \Gamma^{\prime}}{z(\Gamma, t)-z\left(\Gamma^{\prime}, t\right)} & =f d \Gamma^{\prime}\left[\frac{d \Gamma^{\prime}}{z(\Gamma, t)-z\left(\Gamma^{\prime}, t\right)}-\frac{1}{\Gamma-\Gamma^{\prime}}\right] \\
& =-f \frac{d \Gamma^{\prime}}{\left(\Gamma-\Gamma^{\prime}\right)^{2}} \frac{s(\Gamma)-s\left(\Gamma^{\prime}\right)}{1+\frac{s(\Gamma)-s\left(\Gamma^{\prime}\right)}{\Gamma-\Gamma^{\prime}}} \\
& =\int_{\mathcal{C}} \frac{d \Gamma^{\prime}}{\left(\Gamma-\Gamma^{\prime}\right)^{2}} \frac{s(\Gamma)-s\left(\Gamma^{\prime}\right)}{1+\frac{s(\Gamma)-s\left(\Gamma^{\prime}\right)}{\Gamma-\Gamma^{\prime}}}-\mathrm{i} \pi \frac{s_{\Gamma}}{1+s_{\Gamma}},
\end{aligned}
$$

where the path $\mathcal{C}$ is taken for $\Im \Gamma>0$. If $s$ was analytic in the upper half plane, the integral in (17) would vanish, and the motion of the vortex sheet would reduce to the simple equation

$$
s_{t}^{*}=-\frac{1}{2} \frac{s_{\Gamma}}{1+s_{\Gamma}} .
$$


In a second step, it was demonstrated that for a wide class of regular initial conditions of small amplitude, the contour integral in (17) can actually be neglected [7, 8]. Under these conditions, equation (18) contains all the information about the sheet singularity. Numerical simulations showed that the singularity approaches the real axis following the imaginary axis: $\Gamma=\mathrm{i} y, s(\Gamma, t)=$ $f(y, t)$, and $s *(\Gamma, t)=-\bar{f}(y, t)[16,28]$. Therefore, equation (18) together with its complex conjugated, reduces to the local partial differential equations

$$
\bar{f}_{t}=-\frac{\mathrm{i}}{2} \frac{f_{y}}{1-\mathrm{i} f_{y}}, \quad f_{t}=\frac{\mathrm{i}}{2} \frac{\bar{f}_{y}}{1+\mathrm{i} \bar{f}_{y}} .
$$

These are the equations deduced by Moore in 1979 [22]. Using the transformation

$$
1-\mathrm{i} f_{y}=\left(\frac{h \mathrm{e}^{-i g}}{2}\right)^{1 / 2}, \quad 1+\mathrm{i} \bar{f}_{y}=\left(\frac{h \mathrm{e}^{i g}}{2}\right)^{1 / 2}
$$

equations (19) become simpler,

$$
h_{t}=g_{y}, \quad h^{2} g_{t}=h_{y},
$$

and can be solved using the method of characteristics. It is streightforward to find the equations satisfied by the characteristics: (21), $d y / d t= \pm 1 / h=d g / d h$, from which one obtains the two families,

$$
\begin{aligned}
& g-\ln h=a\left(y_{0}\right)=\text { const. } \quad \text { on } \quad \frac{d y}{d t}=\frac{1}{h} \\
& g+\ln h=b\left(y_{0}\right)=\text { const. } \quad \text { on } \quad \frac{d y}{d t}=-\frac{1}{h}
\end{aligned}
$$

where $a$ and $b$ are determined as functions of the characteristic family parameter $y_{0}$, through the initial condition $s(\Gamma, 0)=\mathrm{i} \epsilon \sin (\Gamma)$, or in terms of $h$ and $g$,

$$
h(y, 0)=2+\frac{1}{2} \epsilon^{2} \mathrm{e}^{2 y}, \quad g(y, 0)=-2 \arctan \left(\epsilon \mathrm{e}^{y} / 2\right) .
$$

For small $\epsilon$ an approximation of the characteristics can be found [23]:

$$
y=y_{0}+t / 2+\frac{1}{2} \epsilon \mathrm{e}^{y_{0}}\left(\mathrm{e}^{t}-t-1\right)
$$

and

$$
y=y_{0}-t / 2-\frac{1}{2} \epsilon \mathrm{e}^{y_{0}}\left(\mathrm{e}^{-t}+t-1\right)
$$

This second family possesses an envelope (that marks the trajectory of the singularity in the $(y, t)$ plane)

$$
y=\ln (4 / \epsilon)-t / 2-1-\ln \left(\mathrm{e}^{-t}+t-1\right)
$$

showing that for small $t$ the singularity is far on the $\Gamma$ imaginary axis, but that it approaches the real axis $y=0$ at time $t_{*}$ given by

$$
1+t_{*} / 2+\ln \left(\mathrm{e}^{-t_{*}}+t_{*}-1\right)=\ln (4 / \epsilon)
$$

Recently Moore and Stuart [8], found an exact solution of the system (21), using a convenient choice of the initial condition and the hodograph method, and showed that the estimation of the 


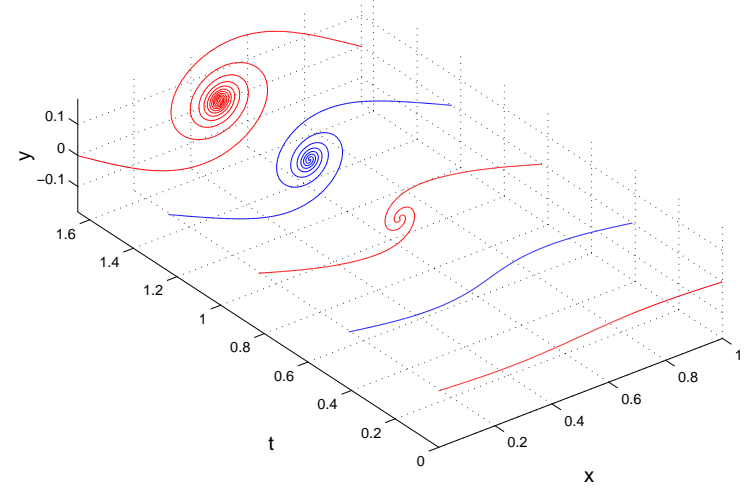

FIG. 4: Numerical computation of the nonlinear evolution of the Kelvin-Helmholtz instability

critical time given by (22) is essentially correct. A more detailed analysis would show that the sheet develops a weak singular inflection point $y \sim \pm|x|^{3 / 2}$ (in some local coordinates of the singular point $(x, y)=(0,0))$, in the region of maximum compression rate. Therefore, the curvature at the singular point diverges as $\kappa \sim|x|^{-1 / 2}$, what may be considered as the seed of the double branched spiral (an assertion not yet demonstrated).

\section{B. The development of a secondary instability}

When the regularized kernel (13) is used, instead of the curvature singularity one observes the formation of a spiral, whose core size is of the order of the cut-off parameter $\delta$, as seen in the sequence of the figure 4 . The spiral forms in the region of maximum compression, this is coherent with the fact that the growth rate of the Kelvin-Helmholtz instability increases with diminishing length scales. To demonstrate this point [1], already discussed by Moore [20, 21], we linearize the Birkhoff-Rott equation around the basic state $z_{K H}(\Gamma, t)$ given by the evolution of the primary instability. We insert $z=z_{K H}+\Delta z$ into (11) to obtain,

$$
\frac{\partial}{\partial t} \overline{\Delta z(\Gamma, t)}=\frac{i}{2 \pi} f d \Gamma^{\prime} \frac{\Delta z(\Gamma, t)-\Delta z\left(\Gamma^{\prime}, t\right)}{\left[z_{K H}(\Gamma, t)-z_{K H}\left(\Gamma^{\prime}, t\right)\right]^{2}} .
$$

If one assumes that the basic state is given, at least locally, by the simple power law form

$$
z_{K H}(\Gamma, t)=A t^{\alpha} \Gamma
$$

where $A$ is a dimensional constant, that describes a sheet locally flat, but stretched in time. Using this expression in (23), and developing $\Delta z \sim a_{k}(t) \exp (i k \Gamma)$ in Fourier series, one deduces a differential equation for the Fourier amplitude of mode $k$,

$$
\ddot{a}_{k}(t)=\frac{\dot{\bar{I}}_{k}}{\bar{I}_{k}} \dot{a}_{k}(t)+\left|I_{k}\right|^{2} a_{k}(t),
$$

where

$$
I_{k}(t)=\left(1 / 2 \pi A^{2} t^{2 \alpha}\right) f\left(1-e^{i k u}\right) d u / u^{2}=|k| / 2 A^{2} t^{2 \alpha},
$$




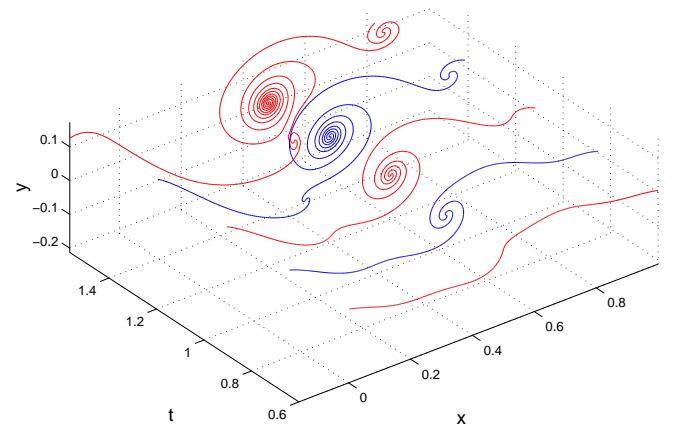

FIG. 5: Growth of secondary vortices from a perturbed vortex sheet. The wave number of the perturbation is $k_{p}=4$, but only two new vortices appear $\left(\delta=0.05, a_{k_{p}}=0.1\right.$, and $\left.t_{0}=0.6\right)$.

and the overdot means time derivative $\left(I_{k}\right.$ may in general be complex, this depends on the form of basic sheet). Note that for $\alpha=0$ one retrieves the Kelvin-Helmholtz growth rate $\sigma_{K H}=\left|I_{k}\right|=$ $|k| / 2 A^{2}$ (with $k=2 \pi n / \lambda$ and $A^{2}=1 / \Delta U$ ). This equation has the same asymptotic properties as the one obtained in Ref. [20]. Equation (24) has an exact solution,

$$
a_{k}(t)=c_{1} \exp \left(\frac{I_{k} t^{1-2 \alpha}}{1-2 \alpha}\right)+c_{2} \exp \left(-\frac{I_{k} t^{1-2 \alpha}}{1-2 \alpha}\right),
$$

showing that (i) for $\alpha<1 / 2$, stretching does not compensate the Kelvin-Helmholtz growth and the perturbation amplitude increases as $a_{k} \sim \exp \left(I_{k} t^{1-2 \alpha} /(1-2 \alpha)\right.$ ); and (ii) for $\alpha>1 / 2$, the amplitude decreases algebraically $a_{k} \sim t^{1-2 \alpha}$. In the $\alpha<1 / 2$, and for fixed $t$, the effective growth rate $\left|\left(1 / a_{k}\right) d a_{k} / d t\right|$ is linear in $k$, as in the primary instability.

Therefore, strong stretching stabilizes the Kelvin-Helmholtz instability, while weak stretching leads to instability, and a fortiori one may assume that compression enhance the vortex sheet instability (the above analysis breaks down for negative $\alpha$ ). This simple argument allows us to think that if the primary unstable vortex sheet is perturbed, the non-uniform stretching may be at the origin of secondary instability and growth of the perturbation amplitude. To investigate this effect, we modified the evolution of the system by introducing at some specific time $t_{0}$ a new perturbation,

$$
z\left(\Gamma, t_{0}\right)=z_{K H}\left(\Gamma, t_{0}\right)+\mathrm{i} a_{k_{p}} \cos \left(2 \pi k_{p} \Gamma\right)
$$

of amplitude $a_{k_{p}}$, wavenumber $k_{p}$, and shifted by $\pi / 2$ in phase, with respect to the initial state. Figure 5 shows a sequence of the evolving vortex sheet, after a $k_{p}=4$ perturbation. Although one may expect that four new spirals form (at the four maxima of the perturbation amplitude, for instance), only two new spirals emerge.

To understand why the other two spirals are smeared out, it is interesting to compute the strain rate $S(s, t)=\boldsymbol{\tau} \cdot \mathbf{V}_{\tau}$ as a function of the sheet length parameter $s=s(\Gamma)$, where $d s^{2}=d x^{2}+d y^{2}$, $\boldsymbol{\tau}(s)$ is the tangent vector at $s$, and $\mathbf{V}_{\tau}$ is the gradient of the velocity in the tangential direction. It appears that $S\left(s, t_{0}\right)$ presents two compression zones just at the formation sites of the two new spirals, the two other ones are placed in stretching zones and are finally damped. Later, as can be seen in figure 6 , the strain rate develops a very complex pattern. The striking difference between the regular shape of the vortex sheet winding and the rich variation of the strain rate, implying a 


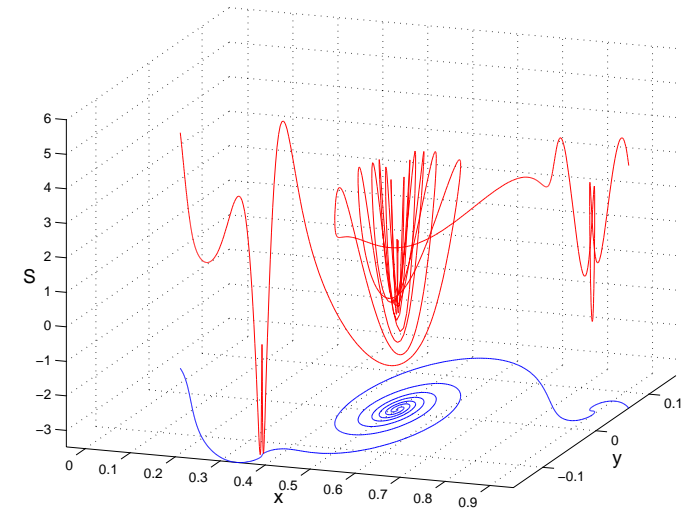

FIG. 6: Stretching of the vortex sheet as a function of position $(t=1)$. The stretching rate is very inhomogeneous even inside the strong central spiral. Compression zones are potentially unstable under Kelvin-Helmholtz instability.

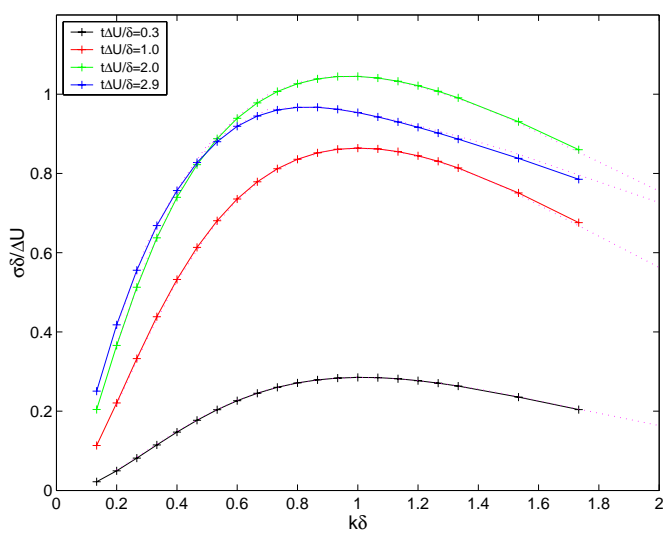

FIG. 7: Dispersion relation of the secondary instability. The growth rate reach an optimal value for a given time. Lengths scale as $\delta$, the cut-off parameter, and times scale as $\delta / \Delta U$ (the initial tangential velocity discontinuity).

complicate vortex intensity function along the sheet, can be related to the "Lagrangian" character of the strain rate at variance to the "Eulerian" character of the vortex sheet motion as a whole. In other words, one may imagine the vortex sheet as formed by a series of point vortices whose motion follows the Hamiltonian dynamics, and tend to concentrate or disperse along the curve in a complicate manner: the local density of these points is a measure of the variation of lengths and at the same time of the local velocity discontinuity (the vortex sheet intensity). The consequences of such a behavior are far reaching, and may be related to the appearance of turbulence [2].

The characterization of the secondary instability is in principle rather involved, because of the complexity of the basic state. However, it appears that the deformation $D(\Gamma, t)=\Im\{z(\Gamma, t)-$ $\left.z_{K H}(\Gamma, t)\right\}$ allows to define a time range so that the Fourier amplitudes $a_{k}(t)$ of the transformed $\hat{D} \equiv \mathcal{F} D$, evolve independently. Therefore, using $\hat{D}(t)$ as a measure of the perturbation, a "linear" evolution time window is found: if initially one puts $a_{k}(0)=a_{k_{p}} \delta_{k, k_{p}}$, with $a_{k_{p}}$ a small constant, the modes with $k \neq k_{p}$ remain small, and the growth of the $k_{p}$ mode can be measured. Indeed, 


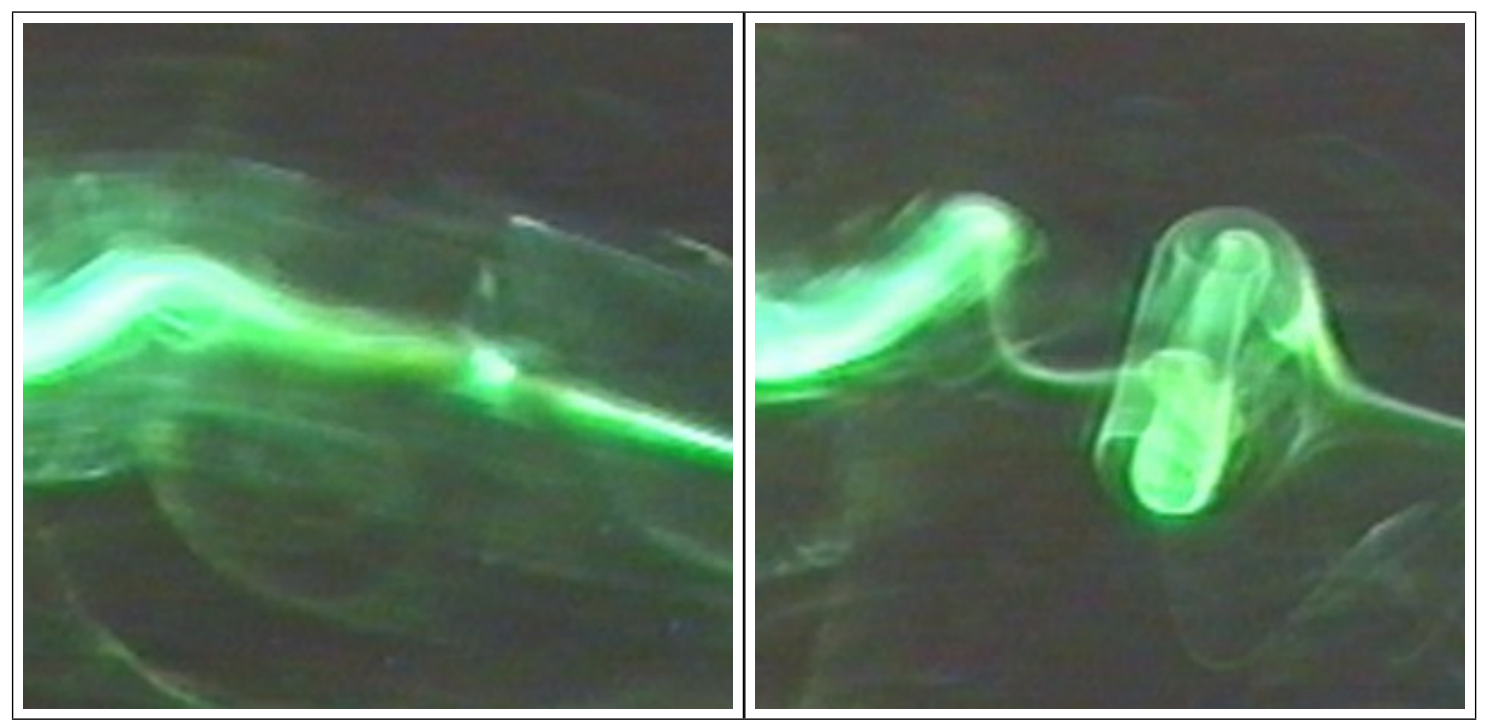

FIG. 8: Vortex breakdown experiment using a triangular plate to generate a conical vortex sheet. This geometry allows for the generation of a vortex having an axial flow. At some moment of the vortex sheet evolution, a stagnation point appears at the vortex core (left), leading to the formation of a recirculation zone, here visualized (right).

in this time interval the growth rate of the perturbation $\sigma(t, k)=d \ln \left|a_{k}(t)\right| / d t$ is a well defined function of the wavenumber and of time. The dispersion relation of the secondary instability is shown in figure 7 . The instability is controlled by the dimensional parameters $\delta$, which fixes the scale of damping (the high wavenumber cut-off), and $\delta / \Delta U$, which fixes the time scale evolution of the growth rate. However, a new time scale appears in the problem, the optimal growth rate, that is the time $t_{M} \approx 2.0 \delta / \Delta U$, for which the growth rate appears to be maximum. The wavenumber of maximum amplification also depends on time, and it tends to diminish as the system evolves rendering unstable much larger scales, an effect related to the elongation of lengths on the vortex sheet [1]. In summary, the secondary instability is intrinsic to the vortex sheet evolution, and not a spurious effect due to external perturbations. Moreover, the nonlinear evolution of the secondary perturbations depends crucially on the local strain rate, whose Lagrangian nature accounts for its complex time evolution. The creation of a fine structure in the vortex sheet properties (strain rate and intensity) as time evolves, makes difficult to predict its long time behavior.

\section{Vortex breakdown in the core of a conical vortex sheet}

Although bi-dimensional vortex sheets have a rich variety of behaviors, some of which are not well understood, such as the stability of their self-similar winding, in three dimensional flows, topological transitions open the way to unexpected effects (see figure 8). Let us consider the flow created by the motion of a triangular plate, instead of the rectangular one that created the vortex sheet we showed in figure 3. When the triangular plate starts to turn, a vortex sheet is generated, that one can loosely compare with the flow around a delta wing. The larger the width of the plate, the larger the circulation, and as a consequence, the more intense the vortex sheet and the 
greater the number of turns in its spiral. Therefore, a vortex sheet with a conical winding shape is released. At the same time, the core of the sheet, that rapidly evolves to a concentrated vortex, is stronger in the wider plate region than in the thinner plate one, and from the relation between vorticity and pressure, a difference of pressure should be generated, and a subsequent axial motion of the fluid from the high pressure region of weak vorticity and the lower pressure in the region of the strong vortex core. The existence of this supplementary axial flow, to the usual rotational one, deeply change the properties of the flow with respect to the two-dimensional case.

The ratio between the characteristic axial velocity $V$ and the characteristic rotational one $U$ is the Rossby number Ro $=V / U$. One may estimate these characteristic velocities from the experimental conditions: $V \sim \Delta p / \rho L$, where $\Delta p$ is the pressure drop and $L$ is the length of the plate, and $U \sim \omega a \sim \Omega R$, where $\omega$ is the core vorticity, and $a$ its size, or in terms of the plate parameters, $\Omega$ the angular velocity and $R$ the plate width. In fact, the axial flow cannot be continued indefinitely because of the finite length of the plate, implying some deceleration region. In the actual experiment the plate is vertical (the wider edge on the top), immersed in water, and the vortex ends at the free surface. Therefore, the axial velocity cannot be a monotonous function of the height $z$. The combination of a small Rossby number and of a deceleration zone is well known to be favorable to the appearance, in stationary flows, of vortex breakdown [5, 26]. Vortex breakdown is characterized by the sudden change in the vortex core, the appearance of a strong deceleration of the axial flow, often followed by the appearance of a stagnation point, and the subsequent increase in the core size, where recirculation zones appear. In figure 8 such a typical situation is presented, but contrary to the usual situation, this experiment demonstrate the vortex breakdown in an open non-stationary flow.

The physical description of the vortex breakdown is a subject of debate [29]. However, from the experiment of figure 8 one can draw some conclusions. Often the vortex breakdown is related to the existence and uniqueness of steady solutions of Euler equations, and is thought to result from a fold bifurcation [6]. Alternatively, some effort was spent to relate vortex breakdown to "shock" formation in the core of slender vortices (vortex filaments) [19], and to a finite time singularity [29]. This second approach seems to be more appropriated to account for the present experiment. We observe vortex breakdown as the normal evolution of the flow, and not as a consequence of the change of some external parameter, as in the case of steady flows in pipes - the usual setup of vortex breakdown experiments. The position where the stagnation point appears is not directly related to some length associated with the deceleration near the free surface (or to the change in curvature of the vortex core, which orients itself perpendicular to the free surface, instead to follow the inclined line of the plate edge). The very appearance of a stagnation point, that is a topological transformation of the basic flow, as time evolves is a manifestation of some singular behavior. Many open questions remain, in particular the influence of the conical structure of the vortex sheet and the associated non-slenderness of the vortex core.

\section{CURVATURE SINGULARITIES OF A DRIVEN FREE SURFACE}

Fluid interfaces are privileged physical systems to visualize and investigate singularity formation: the simple burst of a bubble, the fragmentation of a thread of water, the wave breaking are common examples. These systems, which a first sight may appear as fairly simple, can in fact show very complex behavior. One illustration of the rich dynamics present in simple configurations is the 

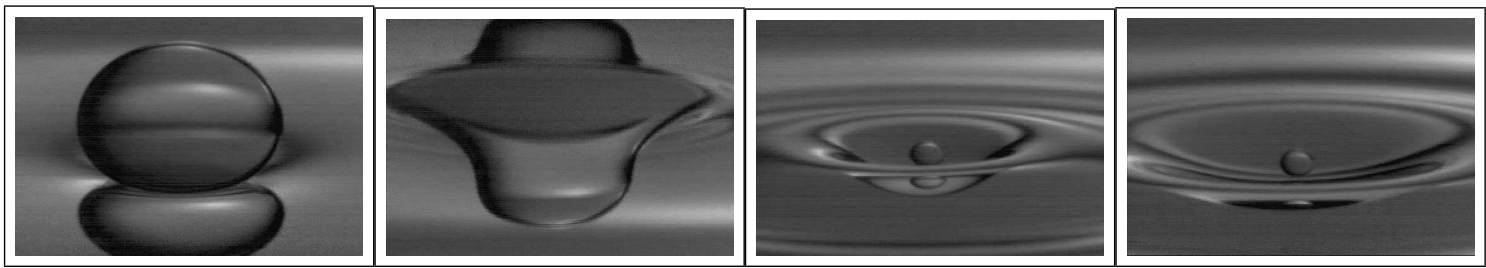

FIG. 9: Sequence showing the burst of a water drop at the oil-water interface, the formation of a cavity, the appearance after collapse of the cavity of a new water drop, and this new drop before its own burst (from left to right). This cycle can repeat several times producing a series of smaller and smaller drops, up to the complete separation of the two immiscible fluids.

oil-water interface shown in figure 9. Initially a drop of water, immersed in the oil phase, lies just above the interface. A thin oil film present between the drop and the interface, slowly drains out, up to the moment for which its two walls touch each other. After the pinching of the oil film the water drop reconnects to the water phase, a cavity opens, as in the similar case of the burst of an air bubble. The retraction of the large cavity is not uniform, and at some moment a change in curvature arises in the bottom region. This change in the bottom curvature is also observed at the air-water interface, for the cavity left by the burst of a bubble, and in this case it results in the emergence of a jet. In the oil-water case what we see is that a new water drop is trapped. The final situation is similar to the initial one, but in a much smaller scale, and the whole process can repeat itself for several generations of drops. One can appreciate the complexity of this process by the simple consideration of the range of spatial and temporal scales present: from the slow dynamics of draining, a lubrication flow in part controlled by gravity, to the grow of the hole determined by surface tension, in passing by the pinching singularity for which microscopic forces of the van der Waals type play a role, one finds a range of $10^{7}$ in length and in time scales. In some sense, this wide range of scales and the global complexity of the phenomenon is comparable in difficulty to fluid turbulence.

\section{A. Two dimensional free surface formalism}

As is the case in turbulence, it is important to study relevant elementary mechanisms in order to understand the physics of singularity formation at interfaces. One interesting situation was observed in the Joseph group with experiments on cusped interfaces [14]. Although their original motivation was the study of the rheology of complex fluids, they found that the free surface of a Newtonian viscous fluid entrained by the motion of a semi-immersed cylinder could form a cusp. This is a particular important observation, which may be compared to the contact line problem: the stress forces diverge at the curvature singularity. Moffatt and Jeong [13] studied the stationary Stokes flow of a viscous fluid near the cusp, and demonstrated that capillary effects are enough to regularize the curvature singularity, but the regularization length is extremely small, even with respect to atomic distances. The curvature radius at the "cusp" is of the order of $\exp (-32 \pi \mathrm{Ca})$, where $\mathrm{Ca}=U \mu / \sigma$ is the capillary number ( $\mu$ the fluid viscosity, and $\sigma$ the surface tension). Even for very small $\mathrm{Ca}$ the curvature is a formidable number.

An interesting related problem is the formation of the cusp singularity, as time evolves, in a free surface driven by a dipole (the geometry introduced in [13], see the figure 10). One may ask 


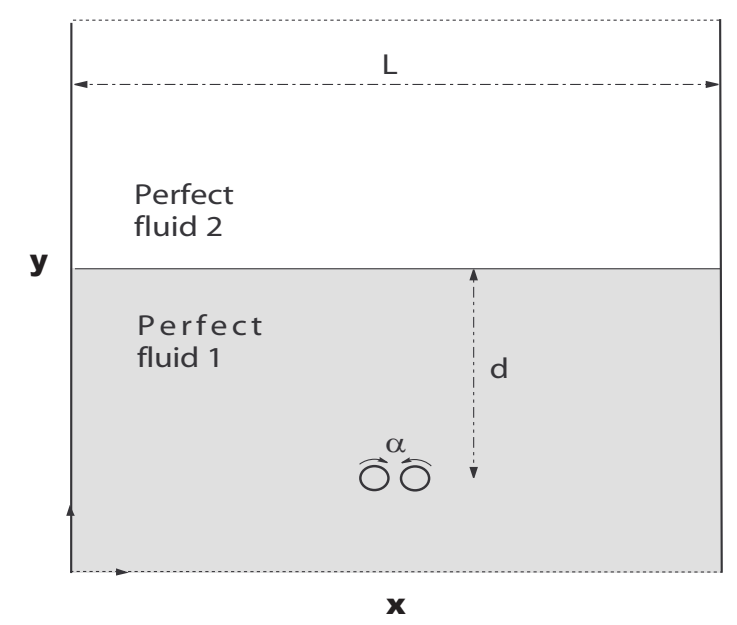

FIG. 10: Problem set-up of a free surface driven by an under beneath dipole. The interface of length $L$ separates two perfect fluids. The interface is advected by a fixed dipole vortex of intensity $\alpha$, placed at a depth $d$. This is a two dimensional problem in the plane $(x, y)$.

whether the cusp, a specific solution of the Stokes flow (a linear stationary problem), can result from the dynamical evolution of an interface (a nonlinear time dependent problem). In its simplest formulation this initial value problem can be studied by neglecting viscous and capillary effects, to focus on inertial effects. The dimensional parameters of the system are: $\alpha$ the dipole strength (it has dimensions $\left.\mathrm{L}^{3} / \mathrm{T}\right), g$ the acceleration of gravity, $d$ the depth of the dipole, $L$ the length of the system (we assume periodic boundary conditions in the $x$-direction) and $\rho_{1}, \rho_{2}$ the densities of the two fluids (we assume in the following that $\rho_{2}=0$ and $\rho_{1}=\rho$ ). Three non-dimensional numbers can be defined to completely characterize the system, the length ratio $D=d / L=0.1$ (for most simulations), and the Atwood number $A=\left(\rho_{1}-\rho_{2}\right) /\left(\rho_{1}+\rho_{2}\right)=1$, which we take fixed, and the Froude number $\operatorname{Fr}=\alpha / \sqrt{d^{5} g}$.

In two dimensions $(x, y)$ the equations describing the free surface motion $y=s(x, t)$, of a perfect fluid, are the Laplace equation for the velocity potential $\Delta \phi=0$, and the boundary conditions at the interface. The Bernoulli equation

$$
\phi_{t}+\frac{1}{2}|\nabla \phi|^{2}+\frac{1}{\mathrm{Fr}^{2}} s(x, t)=0, \quad y=s(x, t),
$$

(in nondimensional form), where we put $p=0$ and the velocity potential includes the contribution of the dipole. The kinematic condition

$$
s_{t}+\phi_{x} s_{x}=\phi_{y}, \quad y=s(x, t)
$$

insures the continuity of the interface motion, the normal velocity of the interface is equal to the normal velocity of a fluid particle attached at the interface.

A more convenient form of these equations can be obtained by conformal transformation $z=$ $x+\mathrm{i} y=z(\zeta, t)$ of the fluid domain $y<s(x, t)$ into the lower half plane $\eta<0$, where $\zeta=\xi+\mathrm{i} \eta$ $[11,27]$. In the $\zeta$-plane the interface is simply given by $\eta=0$, and we may take the dipole placed at $\zeta=-\mathrm{i}$, such that $z(-\mathrm{i}, t)=-\mathrm{i}$. Therefore, the function $z(\zeta, t)$ constitutes a parametric representation of the interface in the original $z$-plane with $x=x(\xi, t)$ and $y=y(\xi, t)$. The complex 
velocity potential $f=\phi+\mathrm{i} \psi=f(\zeta, t)$ is analytic in the lower half plane, except at the dipole position, where $f(z) \sim \mathrm{i} \alpha /(z+\mathrm{i})$.

Using the complex potential to express the fluid velocity $f_{z}=u-\mathrm{i} v \equiv w$ in terms of the new variables $f_{\zeta} / z_{\zeta}=u-\mathrm{i} v$, and noting that the normal at the interface is $\mathbf{n}=\left(-y_{\xi}, x_{\xi}\right)$, the equality of the normal fluid velocity $(u, v) \cdot \mathbf{n}$ and the interface normal velocity $\left(x_{t}, y_{t}\right) \cdot \mathbf{n}$, gives for the kinematic condition in the $\zeta$-plane,

$$
\Im \bar{z}_{t} z_{\zeta}=\Im f_{\zeta}
$$

where the last term includes the dipole contribution. Although this expression is reminiscent to equation (3), in order to compare both equations, the right hand side of (29) on the free surface $\eta=0$ must be computed. To this end, we use the fact that the velocity potential $f$ is harmonic, and then it can directly be determined from the knowledge of the velocity distribution on the interface itself, by the analog of the Birkhoff-Rott equation applied to the fluid normal velocity at the interface (this is in turn a consequence of the Biot-Savart formula):

$$
\left.\Im f_{\zeta}\right|_{\eta=0}=-\frac{1}{\pi} f \frac{\phi_{\xi}\left(\xi^{\prime}\right)}{\xi-\xi^{\prime}} d \xi^{\prime} \equiv-\mathcal{H} \phi_{\xi}
$$

where $\mathcal{H}$ denotes the Hilbert transform. Moreover, we used $\Im w=\psi$ and the property that the imaginary part of the complex potential, which is an analytic function in the lower half plane, can be determined by its real part through the Hilbert transform (see Henrici's book for an account of the Hilbert transform properties [12], vol. 3). The fact that in the transformed variables the free surface is given by the straight line $\eta=0,-\infty<\xi<\infty$, allows this simple expression hold for the velocity at the interface. Then, on the interface we have [32],

$$
\Im \bar{z}_{t} z_{\xi}=-\mathcal{H} \phi_{\xi}
$$

and, at variance to (3), (31) is an integro-differential equation coupled with (27) through the velocity potential. The Bernoulli equation in terms of the transformed variables, takes the form

$$
\phi_{t}+\frac{1}{\operatorname{Fr}^{2}} \Im z(\xi, t)+\frac{1}{2} \frac{\left|f_{\xi}\right|^{2}}{\left|z_{\xi}\right|^{2}}=\frac{1}{\left|z_{\xi}\right|^{2}} \Re\left(\overline{f_{\xi}} \bar{z}_{t} z_{\xi}\right)
$$

on $\eta=0$.

The expression of the Bernoulli equation in terms of quantities computed at the interface is much more cumbersome. It was recently deduced by Zakharov and Dyachenko [9, 32],

$$
\phi_{t}+\frac{1}{\operatorname{Fr}^{2}} \Im z(\xi, t)=\frac{1}{\left|z_{\xi}\right|^{2}} \mathcal{H}\left(\phi_{\xi} \mathcal{H} \phi_{\xi}\right)+\phi_{\xi} \mathcal{H}\left(\frac{\mathcal{H} \phi_{\xi}}{\left|z_{\xi}\right|^{2}}\right),
$$

where one recognizes on the right hand side, the quadratic terms of the velocity, scaled with the Jacobian of the conformal transformation $\left|z_{\xi}\right|^{2}$, which under some circumstances can be large. If the Jacobian was effectively large, in general this assumption is demonstrated a posteriori, the surface potential function can be approximated by $\phi \approx-\lambda^{2} t / 2+\lambda \Im\{z\}+\phi^{D}$, where $\lambda$ is an arbitrary constant, and $\phi^{D}$ is the dipole contribution. Therefore, the "high Jacobian approximation" leads to the Lagrangian growth equation, as the one analyzed in section IB, but with an extra contribution coming from the dipole. This equation has cusped solutions as in the case of the Hele-Shaw problem. 
The simplest case for which the interface evolves towards a cusp is the one where the interface is simply advected by the dipole field. In this case, and for a flat initial condition, an asymptotic solution near the singular point can be obtained from the characteristics of (28),

$$
x_{t}=\psi_{y}, \quad y_{t}=-\psi_{x}
$$

where we used $y=s(x, t)$ and $\psi=\Im \mathrm{i} /(z+\mathrm{i})$ is the current function of the dipole. Therefore, the equation of the characteristics are Hamiltonian, with $\psi$ as the Hamiltonian. As $\psi$ is independent of time, it is itself a constant of motion, a property that permits to reduce (34) to a first order differential equation. The family of characteristics are in principle a function of the two arbitrary constants $\left(x_{0}, y_{0}\right)$, related to the initial point in the interface. We take $\left(x_{0}, 0\right)$ as the initial condition (the flat surface); for such an initial condition the constant "Hamiltonian" is $\psi=x_{0} /\left(x_{0}^{2}+1\right)$. This last condition allows to find $y=y(x, t ; x 0)$, or $x=x(y, t ; x 0)$ in closed form. The singular point $(0,-1)$ is approached following the laws:

$$
x\left(t ; x_{0}\right) \sim x_{0}\left(1-t / t_{0}\right)^{2 / 3},
$$

and

$$
y\left(t ; x_{0}\right)+1=\sqrt{\frac{x_{0}^{2}+1}{x_{0}} x-x^{2}} \sim\left(1-t / t_{0}\right)^{1 / 3},
$$

where $t_{0}=t_{0}\left(x_{0}\right)=x_{0}^{3} / 3\left(x_{0}^{2}+1\right)^{3 / 2}$ (for small $\left.|x|\right)$. The point of symmetry $x=0$ is the first to attain the singular point,

$$
y+1=(1-3 t)^{1 / 3},
$$

which gives $t_{*}=1 / 3$ for the singularity formation time (this is the time at which the interface touches the dipole). The form of the interface at this time is obtained from the expression of $x_{0}$ as a function of $x$ and $t$ in the limit $x \rightarrow 0$, and $t \rightarrow t_{*}$, and substituting into (36),

$$
y+1 \approx|x|^{2 / 7}, \text { at } t=t_{*} .
$$

Equation (38) shows that at $t=t_{*}$ the free surface forms a cusp.

\section{B. Waves, corners and cusps}

The behavior of this simple system is rather rich, and several regimes are observed as the Froude number is changed. In order to investigate the behavior of the interface as a function of the Froude number we perform a series of numerical simulations, using the boundary integral method. The motion of the surface is determined by the equation for the complex potential:

$$
f(z, t)=\frac{1}{4 \pi \mathrm{i}} f_{0}^{2 \pi} \mu(s, t) z_{s}(s, t) \cot (z-z(s, t)) d s+f^{D}(z)
$$

in terms of the distribution of the potential discontinuity on the free surface $\mu(s, t)$, and where $z=z(s, t)$ is a point on the surface; (39) is the analogous of the Birkhoff-Rott equation but instead of the velocity of the sheet, it determines the potential function. The last term $f^{D}(z)=-\mathrm{i} \pi D \tan (\pi D(z+i))$ is the contribution from the dipole, computed at the surface point 
$z$. The $\cot z$ kernel replaces de usual $1 / z$ one, because of the periodic boundary conditions used in numerical computations. Equation (39) is coupled with the surface kinematic condition (28), for the temporal evolution of the interface, and to the Bernoulli equation (27), that allows to find the distribution $\mu$. The interface between two fluids can be assimilated to a surface of discontinuity in the velocity potential (the stream function is continuous across the interface). The quantity $\mu=\phi_{1}-\phi_{2}$ defines then a "dipole intensity", equivalent to the velocity discontinuity of a vortex sheet. We rewrite these equations in terms of the quantities attached to the surface $z=z(s, t)$, $f=f(z(s, t), t)$, and $\mu=\mu(s, t)$ :

$$
\bar{z}_{s} z_{t}(s)=\bar{f}_{s}
$$

is the kinematic condition (compare with (29)), and

$$
\mu_{t}+\frac{\mu_{s}^{2}}{4\left|z_{s}\right|^{2}}=2 \Re\left\{f_{t}\right\}+\frac{\left|f_{s}\right|^{2}+\mu_{s} \Re\left\{f_{s}\right\}}{\left|z_{s}\right|^{2}}-\frac{2}{\operatorname{Fr}^{2}} \Im\{z\},
$$

is the Bernoulli equation for the potential discontinuity. The set $(39,40,41)$ constitutes a complete system that is at the base of the numerical method (see for example [3]).

Schematically, as the Froude number increases, one may distinguish several regimes:

(i) waves, linear and weakly nonlinear stationary waves, range of Froude numbers $\operatorname{Fr} \approx(0,0.1)$;

(ii) corners, formation of cavities whose evolution leads to curvature singularities; these are associated to the appearance of corners, Froude numbers in $\operatorname{Fr} \approx(0.1,0.6)$;

(iii) cusps, formation of cusp singularities when the interface motion is dominated by advection in the dipole velocity field, $\mathrm{Fr}>0.6$.

The (i) regime is related to very small Froude numbers, for which the interface is dominated by the restoring action of gravity, and linear (in the limit $\mathrm{Fr} \rightarrow 0$ ) or nonlinear standing waves are observed. The linearized boundary conditions together with the Laplace equation for the potential, can be analytically solved using Fourier series method, allowing for a comparison with numerics. An interesting phenomenon emerges in the moderate Froude number range, where the regime of non-linear waves breaks down and a new regime characterized by finite time singularities sets in: the interface develops a kind of cavity, whose curvature locally increases up to the appearance of a singularity of the wedge type (figure 11). It is interesting to note that the singularity is approached following a self-similar law, and the angle of the corner results to be independent of Fr (within the given range). The wedge angle is rather flat $\beta \approx 160$, and although this value does not vary with Fr, the size of the region, around the singularity point, where a self-similar evolution of the interface form is observed diminishes with the Fr number. This explains the very existence of a range of Fr where wedge formation is observed, in particular the existence of un upper limit in Froude number above which the corner region size vanishes and the interface evolution change qualitatively. Moreover, the temporal self-similarity of the interface near the singularity can be characterized by the acceleration at the singular point $\left(0, y_{0}\right)$. The acceleration diverges as $\ddot{y}_{0}(t) \sim-1 / \sqrt{-t}$ (for $t \rightarrow 0$, the singularity time), the minus sign comes from the fact that the corner is forming in a ascending surface. This strong deceleration near the singularity is consistent with the behavior of the interface, whose evolution is rather independent of the Froude number: the acceleration due to gravity becomes negligible with respect to the surface acceleration. 

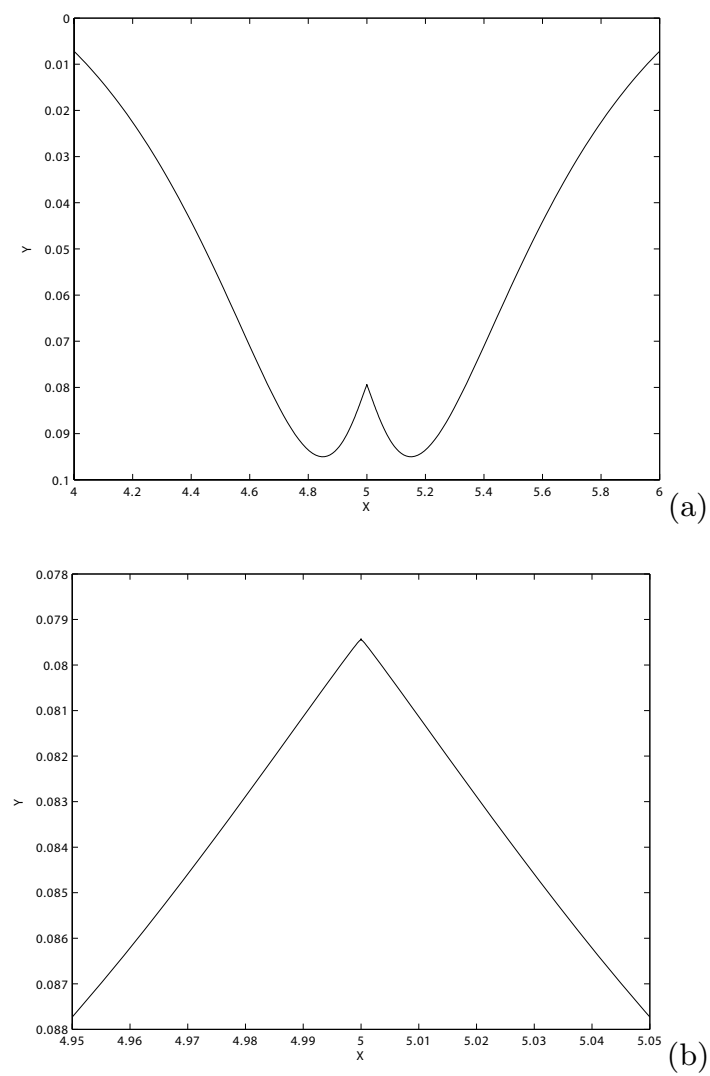

FIG. 11: Formation of a corner for intermediate values of the Froude number (a). Zoom of the singularity region (b). The corner angle is about $160^{\circ}$.

The mechanism of corner formation may be related to the approach of a singular point, of the stagnation velocity type, present in the upper half plane, that is in the non-physical domain, where singularities initially absent can emerge during the system evolution. The formation of the corner, in this scenario, would coincide with the arrival of the stagnation point at the interface. This is supported by the numerical evidence just mentioned: the velocity of the interface tends to zero $\dot{y}_{0}(t) \sim \sqrt{t}$ (or to a constant, depending on the Galilean frame) at the corner together with the fluid velocity, while the deceleration diverges. The appearance of a stagnation point at the fluid interface, allows to speculate about the further evolution of the system. On may think that this is the precursor of the formation of a jet, as often observed in the collapse of cavities after the bubble burst (see [33]). Indeed, the stagnation point entering the fluid must be accompanied, as a result of momentum conservation, by the motion of two opposite jets, one moving towards the bottom, and the other one ascending and deforming the surface. We remark however, that our results concern the two dimensional geometry, and the collapse process (the formation of the wedge results from the converging motion of the fluid) can be much more violent in three dimensions, where the fluid is allowed to converge to a point rather than to a line. Therefore, it would be interesting to investigate, under analogous conditions, the axisymmetric flow, and the formation of a conical singularity as precursor of jet emission.

In the large Froude number limit $(\mathrm{Fr}>0.6)$ the corner is no more observed, the interface is 


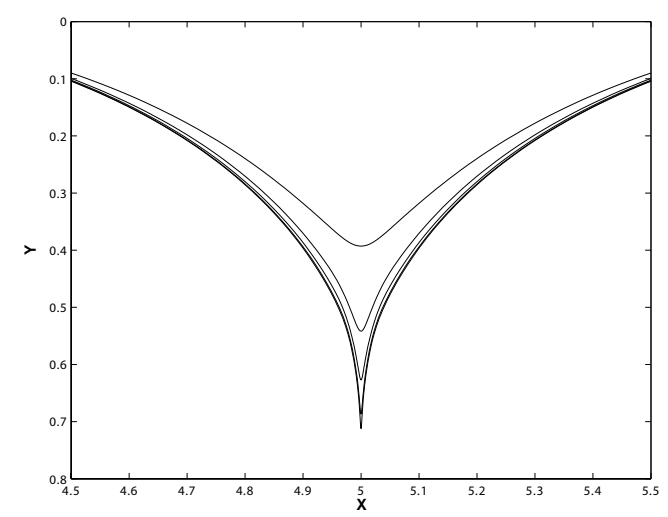

FIG. 12: For large Froude (Fr $>0.6)$ a cusp is formed. The interface is shown for different times, near the singularity. The singular shape of the interface is attained before it reach the dipole $($ at $y=-1)$.

continuously stirred by the dipole towards its direction, and finally a cusp is formed (figure 12). In the case of stationary viscous flow the cusp form $y_{0}(x) \sim|x|^{\alpha}$, is characterized by the exponent $\alpha=2 / 3$, leading to a divergence of the curvature as $\kappa \sim|x|^{-4 / 3}$. For the perfect fluid we found a power law whose exponent depends on the Froude number $\alpha=\alpha(\mathrm{Fr})$. As the Froude number increases it pass from a value of $\alpha \approx 2 / 5$ (for $\mathrm{Fr} \approx 1$ ) to a value of $\alpha \rightarrow 2 / 7$ as $\mathrm{Fr} \rightarrow \infty$ (in practice $\mathrm{Fr} \approx 10$ is enough to detect this regime). The value of $2 / 7$ corresponds to the flow dominated by the dipole, where the interface is purely advected (see equation (38)). In the same regime, when the deepest point of the interface approaches the dipole, its velocity diverges in time as $\dot{y}_{0} \sim t^{-2 / 3}$, while the curvature diverges in time as $\kappa(t) \sim t^{-2}$, in accordance with the laws derived using the pure advection approximation (35-36-37).

\section{WEAK NONLINEAR WAVES AND SHOCKS OF A CAPILLARY FILM}

In the theory of nonlinear waves two opposite problems, the spreading from an initial peak, or the convergence towards the center of a initial bump, may present essentially different behavior. The classical example is the strong explosion problem of Sedov and Taylor, and its "time" reversal version, the implosion problem of a spherical shock (see a complete account of these problems in the Landau [18] or Whitham [30] books). In the expanding case the shock radius $R(t)$, determined be the initial energy $E_{0}$ and ambient gas density $\rho$, is given by the similarity solution $R \sim\left(E_{0} / \rho\right)^{1 / 5} t^{2 / 5}$, as verified by dimensional analysis (here $t \rightarrow \infty$, meaning that this is an asymptotic result). The convergent shock, although from the dimensional analysis point of view is identical to the explosion case, the only dimensional constants also are the initial shock energy and the ambient gas density, does not satisfy a trivial (dimensional) self-similarity law, but a non trivial one, sometimes called of the second type (see Barenblatt book [4], for a thorough discussion of self-similarity and intermediate asymptotics). This means that the shock radius satisfies asymptotically a power law $R \sim(-t)^{\alpha}$, for $-t \rightarrow 0$ and the scaling exponent $\alpha$, different from the one of the divergent shock case (for which $\alpha=2 / 5$ ). The similarity exponent is not determined directly from dimensions but it is related with more subtle symmetry properties of the system (as revealed sometimes by the application of the renormalization group), or with the whole nonlinear structure 


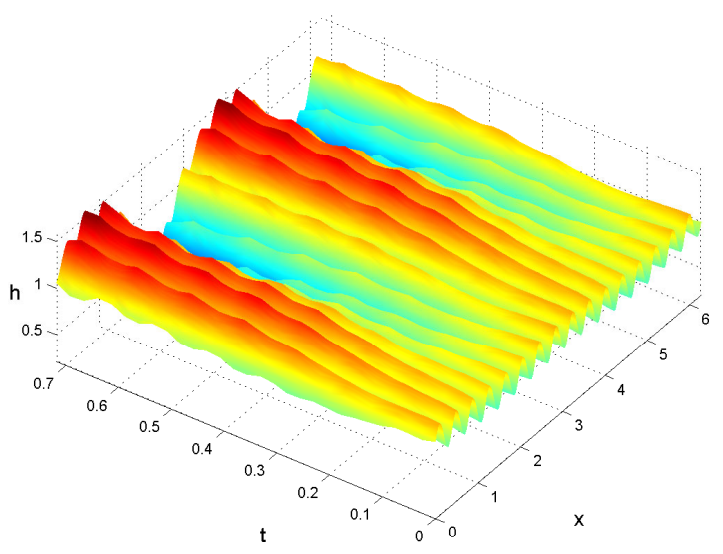

FIG. 13: Modulational instability of a high frequency shallow water capillary wave. Full simulation of the hydrodynamic equations (42) using a spectral method with 512 Fourier modes.

of the equations and boundary conditions (showing that the exponent is in fact not universal, but may change according to the external solution, that is far from the singular point).

One interesting problem of wave convergence is the one related to the fall of a drop on the free surface of a thin film of liquid, resulting in the formation of a splash, a kind of incompressible shock [31]. Waves on a shallow water can be described in the Boussinesq approximation, by mass and momentum conservation in terms of the liquid hight $h(x, t)$ and velocity $u(x, t)$ (in one dimension). In the limit of negligible gravity effects and, for motions driven by inertial and capillary forces, Boussinesq equations reduce to the simple system [30],

$$
h_{t}+(h u)_{x}=0, \quad u_{t}+u u_{x}=S h_{x x x},
$$

where $S=\sigma / \rho$ is the dimensional surface tension to density ratio; using a reference depth $h_{0}$ and $S$ as dimensional parameters, all the quantities can be converted to nondimensional ones: length unit $h_{0}$, time unit $h_{0}^{3 / 2} / S^{1 / 2}$, and velocity unit $\left(S / h_{0}\right)^{1 / 2}$. If one considers mass conservation

$$
Q=\int d x h(x, t)=\text { const. }
$$

one may use $Q^{1 / 2}=h_{0}$ as the definition of the reference length; (43) applies for instance when the initial condition is in the form of a bounded bump. In this case $h_{0}$ does not represent a characteristic length of the system but rather a characteristic amount of matter. The system (42) is for one dimensional waves, the axisymmetric case is readily found to be,

$$
h_{t}+\frac{1}{r}(r h u)_{r}=0, \quad u_{t}+u u_{r}=S\left[\frac{1}{r}\left(r h_{r}\right)_{r}\right]_{r},
$$

and the mass conservation becomes

$$
Q=\int d r r h(r, t)=\text { const. }
$$

where $Q^{1 / 3}=h_{0}$ does not have the same dimensions as in the one dimensional case, and $h=h(r, t)$ and $u=u(r, t)$ depend on the radial coordinate $r$. 


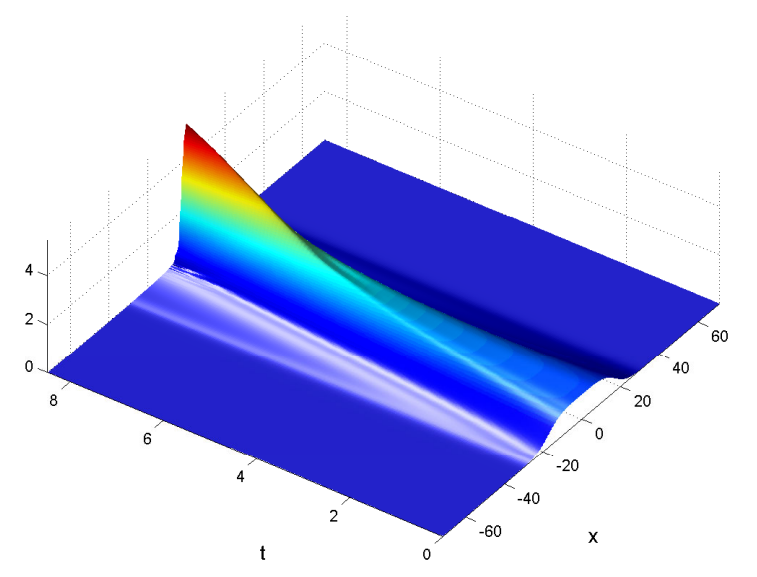

FIG. 14: The shape $h(x, t)$ of the free surface in the case of wave focusing. The initial condition consists of a bounded bump driven by a convergent flow. One observes the evolution towards a high amplitude peak of vanishing size.

It is easy to find the dispersion relation $\omega(k)$ of linear waves related to (43): $\omega^{2}=h_{0} S k^{4}$, showing that waves propagates in both directions with a phase velocity linear in the wavenumber $\sqrt{h_{0} S k}$. The properties of the linear dispersion relation suggest that weakly nonlinear waves are modulationally unstable, and that their envelope amplitude satisfies a nonlinear Schrödinger equation. This is effectively the case, and a standard computation gives

$$
\mathrm{i} A_{t}+\frac{1}{2} A_{x x}+\frac{7 k^{2}}{4}|A|^{2} A=0,
$$

(in nondimensional form), where $h(x, t)=A(x, t) \mathrm{e}^{\mathrm{i} k x-\mathrm{i} \omega t}$ is the primary wave packet with $A$ a slowly varying amplitude (figure 13). The numerical simulation of (42), using an initial profile consisting of a superposition of a sinusoidal wave with $k=16$, and other one of smaller amplitude with $k=1$ to modulate the first one, shows that for initial weak waves, the system evolves like an almost integrable system (as described by the nonlinear Schrödinger equation). This can be confirmed from the long time behavior of the wave, and observing that the system is quasi-recursive. However, the generic behavior of the system is very far from the one of an integrable one, and for finite initial amplitude waves, the appearance of strong nonlinear effects such as the formation of shocks is systematically observed (figure 14).

In order to understand the behavior of the free surface in the region of singularity formation, we analyze the similarity solutions of (42), subject to the constraint (43). The self-similar solution has in one dimension the form,

$$
h(x, t)=\frac{H(\xi)}{t^{2 / 5}}, u(x, t)=\operatorname{sgn}(t) \frac{U(\xi)}{t^{3 / 5}}, \xi=\frac{x}{t^{2 / 5}},
$$

or in the axisymmetric case,

$$
h(r, t)=\frac{H(\xi)}{t^{2 / 3}}, u(r, t)=\operatorname{sgn}(t) \frac{U(\xi)}{t^{2 / 3}}, \xi=\frac{r}{t^{1 / 3}},
$$

where the presence of $\operatorname{sgn}(t)$ express the time reversal symmetry of the original equations. Indeed, the system is invariant under the transformation $t \rightarrow-t$ and $u \rightarrow-u$, hence equations (47) or (48) 


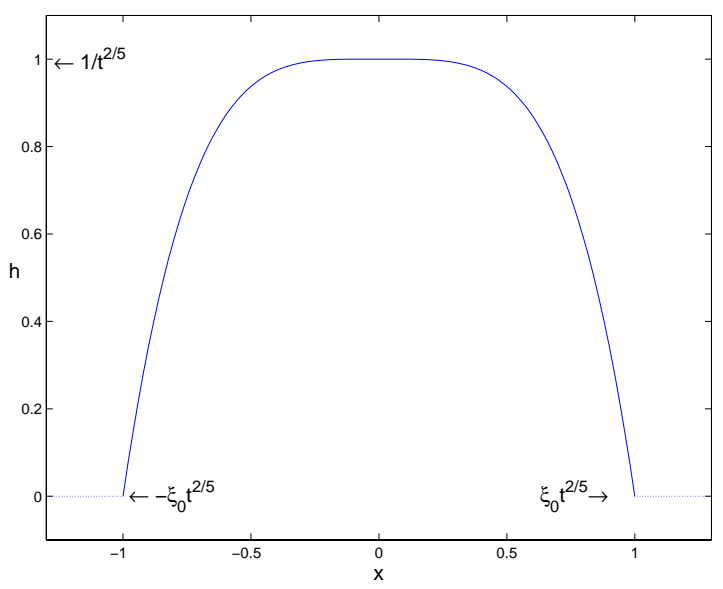

FIG. 15: Self-similar solution of the wave focusing problem in one dimension. Collapse occurs at $t=0$. The wave amplitude increases as $\sim 1 / t^{2 / 5}$ and its width decreases as $\sim 1 / t^{2 / 5}$ (with $t<0$ ).

describe the evolution of the system from an initial concentrated wave packet or the convergence of an initially extended bump.

When equations (47) or (48) are inserted into the evolution equations for one dimensional and axisymmetric waves respectively, one obtains ordinary differential equations that can be solved explicitly in the bounded case. Assuming that the velocity field as well as the liquid height vanish outside a bounded region, it is possible to find a solution, asymptotically valid near the singularity, which remains bounded for all times, and satisfy exactly the original dynamical equations. One may consider that initially this region is at infinity in the focusing case; or concentrated on a point in the expanding case. This situation is equivalent to the case of the Burgers equation in the limit of vanishing kinematic viscosity $\nu$. A self-similar solution can be obtained that coincides with the regular solution in the limit $\nu \rightarrow 0$. In fact there exists an interesting and deep link between self-similar and "weak" solutions of partial differential equations. The one dimensional solution reads,

$$
H(\xi)=\frac{1}{8}\left(\frac{50}{S}\right)^{1 / 5}\left(1-\frac{\xi^{4}}{\xi_{0}^{4}}\right), \quad U(\xi)=\frac{2}{5} \xi
$$

where $\xi_{0}=(125 S / 2)^{1 / 5}$. This solution is depicted in figure 15 . In the case of axisymmetric waves the solution reads,

$$
H(\xi)=\frac{1}{4}\left(\frac{4}{S}\right)^{1 / 3}\left(1-\frac{\xi^{4}}{\xi_{0}^{4}}\right), \quad U(\xi)=\frac{1}{3} \xi
$$

where $\xi_{0}=(432 S)^{1 / 6}$. In both one-dimensional and axisymmetric cases, outside the region $\xi<\left|\xi_{0}\right|$, the self-similar height $H$ and velocity $U$ are zero: the velocity varies linearly $U \sim-\xi$ inside $\xi<\left|\xi_{0}\right|$ and is discontinuous at $\xi_{0}$ (converging flow), while the surface height peaks at $\xi=0$. This behavior is similar to the usual case of a shock in the framework of the simple wave equation $u_{t}+u u_{x}=0$ for the velocity $u$, mentioned before as the inviscid limit of the Burgers equation. In this case it is straightforward to verify the solution $u(x, t)=x / t$ for $x<\sqrt{2 Q t}$ and zero outside, where $Q=\int u d x$; or in self-similar form $u=\sqrt{2 Q / t} U(\xi)$ with $\xi=x / \sqrt{2 Q t}$, and $U=\xi$ for $0<\xi<1$ 
and zero outside (note that here the scaling $x \sim \sqrt{t}$ is also appropriated to the viscous extension of the simple wave equation, that adds a term $\nu u_{x x}$ to the right hand side).

Therefore, bounded regions of converging waves focus to a line (in one dimension) or to a point (in the axisymmetric case). This behavior has been invoked to explain some features of splash formation, after the impact of a drop onto a film of liquid [31]. Moreover, other types of singular behavior may develop in the framework of the Boussinesq system (42). For instance, in the case of a thin film and for a set of initial conditions, the film height can vanish in finite time $h(x, t) \rightarrow 0$, at some point $x_{*}$ and at some instant $t_{*}$ [25], following a self-similar law $h \sim$ $\left(t_{*}-t\right)^{\alpha} H\left(\left(x-x_{*}\right) /\left(t_{*}-t\right)^{\beta}\right)$ near $\left(x_{*}, t_{*}\right)$ (with $\alpha$ and $\beta$ positives). When introducing this form, and a similar one for the velocity, into the Boussinesq equations, a one parameter family of similarity exponents is obtained. The selection of one such a laws can be obtained if one imposes as a constraint that the process of film pinching conserves the energy:

$$
E=\frac{1}{2} \int d x\left(S h_{x}^{2}+h u^{2}\right)
$$

In such a case a self-similar law with $\alpha=2 / 7$ and $\beta=4 / 7$ is obtained. The general case, not yet solved, may present a selection of the similarity law by more subtle mechanisms, as for instance through a solvability condition or for satisfying some external solution, as often encountered in second type similarity laws.

\section{CONCLUSIONS}

Let us mention in conclusion some interesting problems worth to be investigated. Many examples of singularity approach obeying a self-similar evolution are well known, and the subtle mechanism of selection of the power law exponent rather well understood. However, the continuation of the singular solution through the topological transition, continuation that may involve some controlled regularization method, is much less studied, and in parallel much more difficult to analyze, especially when the system also evolves towards a singular configuration. This is precisely the case of the Moore singularity and the formation of the spiral vortex: before the transition the system consists in a Kelvin-Helmholtz unstable vortex sheet approaching an infinite curvature shape, a "cusp", while after the singularity another self-similar regime, the rolling-up of the spiral, sets in. Both states, before and after the transition, can be considered as weak solutions of the Euler equations, or as the zero viscosity limit of the Navier-Stokes equations. Is in this last framework that the problem acquires all its significance. This is also the case of the wedge-jet transition at the interface of two perfect fluids, case that can be considered as being the zero surface tension and viscosity limit of real fluids. Related problems to the singular to singular solution transition, are the stability of self-similar evolutions, their ability to "attract" a wide range of initial or boundary conditions, and the status of singularities with respect to smoothing effects (dissipation, capillarity, etc.). A specific problem combining these items, is the stability of vortex sheets when the vortex intensity is inhomogeneous, or equivalently, when the strain rate is variable (the above mentioned Moore singularity is a special case, applied to the initial flat sheet).

Therefore, a central question arises when considering these problems: the relation between dissipation and singular solutions. We saw in the case of focusing of capillary waves that the bounded initial bump amplifies up to infinite amplitude. This process, which is mass conserving 
(the constraint that determined the set of similarity variables), do not conserve energy: the height of the film is not a differentiable function. Although in some cases, as for the Burgers equation or for one-dimensional shocks in a compressible medium, the link relating the self-similar solution with the smoothed solution can be established, in general this relation is unknown. Onsager in 1949, introduced the concept of dissipative solutions of conservative systems [24]. Dissipative solutions are in fact weak or singular solutions of reversible dynamical systems, the ones holding at both sides of topological transitions, or the ones related to finite time singularities. Indeed, these singular solutions are unavoidable when one wants to relate the properties of a conservative system to its associated dissipative counterpart, like Euler equations for a perfect fluid with respect to Navier-Stokes equations for a viscous one (the example taken by Onsager in relation to the turbulent cascade). The investigation of this relation in specific systems may contribute to the understanding of the behavior of physical systems in the limit of vanishing dissipation, a limit often characterized by very complex motions (like in the case of turbulence).

\section{Acknowledgments}

This paper is the result of a cooperative research work. The vortex sheets and vortex breakdown results were obtained in collaboration with Malek Abid (instability of a stretched vortex sheet) and Thomas Leweke, Patrice Meunier and Christophe Lepage (experiments with plates). The stirred free interface problem is part of the thesis work of François Grimal. The work on the capillary shock is the fruit of a collaboration with Thomas Frisch. I thanks all of them. Discussions with Patrice Le Gal, Stéphane Le Dizès and Emmanuel Villermaux are acknowledged. I am also glad to thanks Enrique Tirapegui and Sergio Rica for their kind invitation to participate at the Viña del Mar meeting.

[1] M. Abid and A. D. Verga. Stability of a vortex sheet roll-up. Phys. Fluids, October(10):pp-pp, 2002.

[2] M. Abid and A. D. Verga. Vortex sheet dynamics and turbulence. in preparation, June 2002.

[3] Gregory R. Baker, Daniel I. Merion, and Steven A. Orszag. Generalized vortex methods for free-surface flow problems. J. Fluid Mech., 123:477-501, 1982.

[4] G. I. Barenblatt. Scaling, self-similarity, and intermediate asymptotics. Cambridge University Press, Cambridge, United Kingdom, 1996.

[5] G. K. Batchelor. An Introduction to Fluid Mechanics. Cambridge University Press, London, 1967.

[6] J. D. Buntine and P. G. Saffman. Inviscid swirling flows and vortex breakdown. Proc. R. Soc. London A, 449:139-153, 1995.

[7] R. E. Caflisch and O. F. Orellana. Long time existence for a slightly perturbed vortex sheet. Comm. Pure Appl. Math., 39:807-838, 1986.

[8] Stephen J. Cowley, Greg R. Baker, and Saleh Tanveer. On the formation of Moore curvature singularities in vortex sheets. J. Fluid Mech., 378:233-267, 1999.

[9] A. I. Dyachenko, E. A. Kuznetsov, M. D. Spector, and V. E. Zakharov. Analytical description of the free surface dynamics of an ideal fluid (canonical formalism and conformal maping). Phys Letters A, 221:73-79, 1996.

[10] M. C. Escher. The Graphic Work of M. C. Escher. Pan Ballentine, London, 1961.

[11] Malcolm Grant. Standing Stokes waves of maximum height. J. Fluid Mech., 60:593-604, 1973. 
[12] P. Henrici. Applied and Computational Complex Analysis. Wiley, New York, 1993.

[13] Jae-Tack Jeong and H. K. Moffatt. Free-surface cusps associated with flow at low Reynolds number. J. Fluid Mech., 241:1-22, 1992.

[14] Daniel D. Joseph, John Nelson, Michael Renardy, and Yukiro Renardy. Two-dimensional cusped interfaces. J. Fluid Mech., 233:383-409, 1991.

[15] Robert Krasny. Desingularization of periodic vortex sheet roll-up. J. Comp. Phys., 65:292-313, 1986.

[16] Robert Krasny. A study of singularity formation in a vortex sheet by the point-vortex approxiamtion. J. Fluid Mech., 167:65-93, 1986.

[17] Robert Krasny. Vortex sheet computations: roll-up, wakes, separation. Lectures in Applied Mathematics, 28:385-402, 1991.

[18] L. Landau and E. Lifchitz. Mécanique des Fluides. Mir, Moscou, 1989.

[19] T. S. Lundgren and W. T. Ashurst. Area-varying waves on curved vortex tubes with application to vortex breakdown. J. Fluid Mech., 200:283-307, 1989.

[20] D. W. Moore and R. Griffith-Jones. The stability of an expanding vortex sheet. Mathematika, 21:128$133,1974$.

[21] D. W. Moore. The stability of an evolving two-dimensional vortex sheet. Mathematika, 23:35-44, 1976.

[22] D. W. Moore. The spontaneous appearance of a singularity in the shape of an evolving vortex sheet. Proc. R. Soc. London A, 365:105-119, 1979.

[23] D. W. Moore. Numerical and analytical aspects of Helmholtz instability. In F. I. Niordson and N. Olhoff, editors, Theoretical and Applied Mechanics, pages 263-274. Elsevier, North-Holland, 1985.

[24] L. Onsager. Statistical hydrodynamics. Nuovo Cimento Suppl., 6:279-287, 1949.

[25] M. C. Pugh and M. J. Shelley. Singularity formation in thin jets with surface tension. Comm. Pure Appl. Math., 51:733-795, 1998.

[26] P. G. Saffman. Vortex Dynamics. Cambridge University Press, London, 1992.

[27] L. W. Schwartz. A semi-analytic approach to the self-induced motion of vortex sheets. J. Fluid Mech., 111:475-490, 1981.

[28] M. Shelley. A study of singularity formation in vortex-sheet motion by spectrally accurate vortex method. J. Fluid Mech., 244:493-526, 1992.

[29] M. O. Souza and S. J. Cowley. On incipient vortex breakdown. submitted to J. Fluid Mech., January 2002.

[30] G. B. Whitham. Linear and Nonlinear Waves. Wiley, New York, 1974.

[31] A. L. Yarin and D. A. Weiss. Impact of drops on solid surfaces: Self-similar capillary waves, and splashing as a new type of kinematic discontinuity. J. Fluid Mech., 283:141-173, 1995.

[32] V. E. Zakharov and A. I. Dyachenko. High-Jacobian approximation in the free surface-dynamics of an ideal fluid. Physica D, 98:652-664, 1996.

[33] B. W. Zeff, B. Kleber, J. Fineberg, and D. P. Lathorp. Singularity dynamics in curvature collapse and jet eruption on a fluid surface. Nature, 403:401-404, January 2000. 